\title{
Dopamine-dependent cAMP dynamics in basal amygdala glutamatergic neurons
}

Andrew Lutas ${ }^{1, *}$, Kayla Fernando ${ }^{1}$, Stephen X. Zhang ${ }^{1}$, Abhijeet Sambangi', and Mark L. Andermann ${ }^{1, *}$

${ }^{1}$ Division of Endocrinology, Metabolism, and Diabetes, Beth Israel Deaconess Medical Center, Harvard Medical School, Boston, MA, USA, 02115

* Correspondence:

Andrew Lutas, alutas [at] bidmc.harvard.edu

Mark Andermann, manderma [at] bidmc.harvard.edu 


\section{Summary}

41 Dopaminergic inputs to basal amygdala (BA) instruct learning of motivational salience. Here, we

42 investigated the dynamics of dopamine release and downstream signaling during multiple salient

43 events occurring within tens of seconds. We established in vitro and in vivo real-time tracking and

44 manipulation of CAMP - a key intracellular plasticity signal downstream of dopamine receptor

45 activation. Optogenetically-evoked release of dopamine drove proportional increases in cAMP in

46 almost all BA glutamatergic neurons, suggesting widespread actions of dopamine across neurons

47 preferring positive or negative valence. These cAMP responses decayed more slowly than

48 dopamine release, potentially extending the window of plasticity. cAMP levels accumulated

49 following direct photostimulation of cAMP but not repeated stimulation of dopamine axons, due to

50 potent depression of dopamine release. cAMP and protein kinase A (PKA) responses to repeated

51 appetitive or aversive stimuli also exhibited pronounced depression. Thus, history-dependent

52 dynamics of dopamine and cAMP may regulate learning of temporally clustered, salient stimuli. 


\section{Introduction}

The basolateral amygdala is critical for learning the valence of initially neutral sensory cues and guiding decisions to approach or avoid such cues (O'Neill et al., 2018; Zhang and Li,

57 2018). The neural plasticity that occurs during this associative learning involves dopamine,

58 norepinephrine and other neuromodulators that bind to receptors on target cells to regulate

59 cyclic adenosine monophosphate (cAMP) levels and synaptic plasticity (Bissière et al., 2003;

60 Johansen et al., 2014; Tronson et al., 2006). During associative plasticity, the precise timing of

61 dopamine-dependent cAMP signals is important (Handler et al., 2019; Steinberg et al., 2013;

62 Yagishita et al., 2014). However, we understand much less about the dynamics of dopamine-

63 evoked cAMP signals, which also depend on regulatory mechanisms that vary across states

64 and across neuron types.

An increase in release of dopamine from VTA neurons projecting to the basal amygdala

(BA) occurs during salient events of both positive and negative valence (Lutas et al., 2019).

67 Despite receiving this common dopamine signal, individual BA glutamatergic neurons

68 preferentially respond to either appetitive or aversive events (Lutas et al., 2019; O'Neill et al.,

69 2018; Zhang and Li, 2018). These observations led us to hypothesize that dopamine release

70 broadly facilitates plasticity by increasing cAMP levels in both appetitive- and aversive-preferring

71 BA neurons during salient outcomes. Meanwhile, learning of the valence of the outcome

72 associated with a cue may be achieved via other mechanisms involving calcium-dependent

73 processes driven by distinct pathways relaying information about sensory cues and about

74 positive and negative outcomes (Correia and Goosens, 2016).

We developed an approach to optogenetically control dopamine release from VTA axons

76 in BA (VTA ${ }^{\mathrm{DA} \rightarrow \mathrm{BA}}$ ) while simultaneously visualizing calcium or cAMP dynamics in BA

77 glutamatergic neurons. To first test the sufficiency and timing-dependence of dopamine-evoked

78 cAMP in instructing stimulus salience, we performed longitudinal imaging of calcium responses

79 in BA neurons in awake mice across sessions in which one of two visual stimuli is paired with 
optogenetic stimulation of VTA ${ }^{\mathrm{DA} \rightarrow \mathrm{BA}}$ axons. We found that optogenetic stimulation of dopamine

81 axons was sufficient to drive the acquisition of stimulus responses in a subset of BA neurons

82 across days, similar to the acquisition of responses to cues paired with natural appetitive and

83 aversive outcomes (Lutas et al., 2019). We then investigated the immediate consequences of

84 dopamine receptor activation on BA glutamatergic neurons by tracking intracellular cAMP

85 production using a genetically-encoded fluorescent biosensor (Tewson et al., 2016). We found

86 that exogenous dopamine and transient photostimulation of VTA ${ }^{\mathrm{DA} \rightarrow \mathrm{BA}}$ axons elevated cAMP in

87 most BA glutamatergic neurons. The proportion of BA neurons with dopamine-evoked increases

88 in cAMP scaled with dopamine concentration, and all BA neurons showed elevated cAMP in the

89 presence of high levels of exogenous dopamine. These findings suggest that while dopamine

90 release does not determine the encoded valence of the conditioned stimulus, it may determine

91 its salience by controlling the number of stimulus-responsive BA neurons.

We also found that transient dopamine release triggered cAMP increases lasting for over

9330 seconds. This duration was primarily determined by cell-autonomous processes rather than

94 by prolonged elevations in extracellular dopamine, since we could replicate the cAMP decay

95 kinetics by circumventing dopamine receptors using a photoactivatable adenylate cyclase.

96 Further, we found that presynaptic depression at VTA ${ }^{\mathrm{DA} \rightarrow \mathrm{BA}}$ terminals limits the duration of

97 elevated CAMP when dopamine release events are spaced tens (but not hundreds) of seconds

98 apart. This depression in cAMP signaling was observed for repeated optogenetically-evoked

99 dopamine release as well as for repeated delivery of unexpected palatable food or aversive tail

100 shock. We also observed potent depression at the next stage in the signaling pathway, protein

101 kinase A (PKA) activity, in vivo in response to repeated salient events. Thus, the learned

102 salience associated with novel stimuli could be regulated by the magnitude of dopamine-evoked

103 cAMP within the BA. Together, our results suggest that dopamine-evoked cAMP initiates a

104 widespread permissive plasticity window that is correlated with stimulus salience and engages 
other intracellular signals in BA to control the acquisition of sensory responses to cues surrounding motivationally salient outcomes.

\section{Results}

109 Pairing visual cues with optogenetic stimulation of VTA ${ }^{\mathrm{DA} \rightarrow \mathrm{BA}}$ axons results in cue-evoked 110 calcium responses in BA neurons

112 visual cues when these cues are paired with salient appetitive or aversive outcomes (Lutas et

113 al., 2019; O’Neill et al., 2018; Schoenbaum et al., 1999). To determine whether pairing

114 dopamine release with an arbitrary visual stimulus was sufficient for BA neurons to acquire

115 responses to this stimulus, we combined two-photon calcium imaging of the same individual BA

116 neurons across several sessions with photostimulation of VTA ${ }^{\mathrm{DA} \rightarrow \mathrm{BA}}$ axon terminals

117 (Supplementary Figure 1A,B). We used two simple visual stimuli differing only in the orientation

118 of a drifting grating. One of the stimuli ("Cue A") was paired with photostimulation of VTA DA $\rightarrow$ BA

119 axon terminals, which occurred 200 ms after the visual stimulus offset, and the second ("Cue

120 B") was not paired with any outcome (Supplementary Figure 1C). An inter-trial interval (ITI) of 6

121 seconds was used to mirror our prior study that paired these visual stimuli with salient appetitive

122 or aversive outcomes (Lutas et al., 2019). We first imaged BA neuron responses to the

123 presentation of these visual stimuli prior to any manipulations. Only a small fraction of BA

124 neurons was significantly responsive ( $1 \% ; n=14 / 1283$ neurons from 7 mice) to the presentation

125 of these arbitrary visual stimuli (Supplementary Figure 1D), consistent with prior work (Lutas et

126 al., 2019). We then paired "Cue A" with brief photostimulation of VTA ${ }^{\mathrm{DA} \rightarrow \mathrm{BA}}$ axon terminals

127 across several daily sessions while tracking calcium responses in the same BA neurons. After

128 three days of pairing, a larger fraction of BA neurons had become responsive to the paired

129 visual stimulus (4\%; $n=48 / 1199$ neurons from 6 mice; Supplementary Figure 1D). Despite the

130 randomized nature of stimulus presentations and the six-second ITI between trials, BA neurons 
131 that acquired responses to "Cue A" also acquired responses to the unpaired "Cue B"

132 (Supplementary Figure 1E,F). As discussed below, such generalization may relate to sustained 133 effects of transient VTA ${ }^{\mathrm{DA} \rightarrow \mathrm{BA}}$ axon photostimulation. Nevertheless, responses to "Cue B" were

134 weaker than those to "Cue A", and neurons preferentially responded to "Cue A" rather than "Cue 135 B" (Supplementary Figure 1G,H). Therefore, photostimulating VTA ${ }^{\mathrm{DA} \rightarrow \mathrm{BA}}$ axons immediately

136 following an arbitrary visual cue leads to the acquisition of cue-evoked responses in a subset of

137 BA glutamatergic neurons.

A CAMP sensor reveals spatially broad dopamine signaling across all BA glutamatergic neurons

To better understand the immediate effects of photostimulating VTA ${ }^{\mathrm{DA} \rightarrow \mathrm{BA}}$ axons on 142 postsynaptic neurons, we expressed a Cre-dependent, compact fluorescent biosensor for cAMP, 143 cADDis (Tewson et al., 2016), in BA glutamatergic neurons via AAV injections into EMX1-Cre 144 transgenic mice (Figure $1 \mathrm{~A}, \mathrm{~B}$ ). We tested the sensor functionality by applying the adenylate 145 cyclase (AC) activator forskolin (Figure 1C) or dopamine (Figure 1D) to brain slices to stimulate 146 intracellular cAMP production. Both forskolin and dopamine generated reliable decreases in mean

147 fluorescence intensity, indicating increasing cAMP concentration (Tewson et al., 2016) (note that 148 for visualization purposes, y-axes have been inverted so that a rise in cADDis signal reflects an 149 increase in cAMP; Figure 1C).

We next used cADDis to estimate the fraction of BA glutamatergic neurons that could 152 respond to dopamine. Since almost all BA glutamatergic neurons express Type 1 dopamine 153 receptors (D1) (Lutas et al., 2019; Namburi et al., 2015; O’leary et al., 2020), while only few 154 express Type 2 dopamine receptors (D2), dopamine should elevate cAMP in BA neurons. To 155 directly determine the fraction of BA glutamatergic neurons that showed increases in cAMP in 156 response to dopamine, we extracted fluorescence traces from the somatic regions of individual 
neurons (Figure 1E; see Methods). We first analyzed responses to application of forskolin as a measure of dopamine receptor-independent cAMP production. Forskolin resulted in cAMP 159 increases in all BA glutamatergic neurons. We then found that application of a high concentration 160 of dopamine $(20 \mu \mathrm{M})$ potently elevated cAMP in all BA glutamatergic neurons, similar to forskolin, 161 while lower concentrations drove weaker yet detectable increases in BA neurons (Figure 1F). This

162 finding demonstrates a near-universal potential for dopamine signaling in BA glutamatergic 163 neurons, and suggests that the diversity of valence preferences of nearby BA glutamatergic 164 neurons (Lutas et al., 2019; O'Neill et al., 2018) may not to be due to differences in their ability to 165 increase (or decrease) their cAMP levels in response to dopamine.

Photostimulation of $\mathrm{VTA}^{\mathrm{DA} \rightarrow \mathrm{BA}}$ axons transiently elevates cAMP in almost all BA glutamatergic neurons

While exogenous application of dopamine revealed a widespread increase in cAMP levels

170 in BA glutamatergic neurons, physiological release of dopamine directly from axon terminals may

171 be more spatially restricted. To evoke endogenous dopamine release, we targeted expression of

172 a red-shifted channelrhodopsin (Chrimson) in VTA dopamine neurons using DAT-Cre transgenic

173 mice (Bäckman et al., 2006; Klapoetke et al., 2014). To confirm that photostimulating VTA ${ }^{\mathrm{DA} \rightarrow B A}$

174 axons in brain slices reliably evoked dopamine release, we combined widefield photostimulation

175 of Chrimson with two-photon imaging of a green fluorescent biosensor for dopamine, dLight1.1,

176 that was expressed in BA glutamatergic neurons (Patriarchi et al., 2018) (Supplementary Figure

$1772 \mathrm{~A})$. Brief trains of optical stimulation $(20 \mathrm{~Hz} ; 5 \mathrm{~s}$ duration) generated transient elevations in

178 dopamine that lasted for several seconds (Supplementary Figure 2B). Importantly, fluorescence

179 changes in this D1-based dopamine sensor could be completely blocked by application of a D1

180 antagonist (SCH23390, $300 \mathrm{nM}$, Supplementary Figure $2 \mathrm{C})$, confirming that the signals were

181 mediated by dopamine release and not by artifacts related to optical stimulation. By comparing

182 endogenous release of dopamine following optical stimulation of VTA ${ }^{\mathrm{DA} \rightarrow \mathrm{BA}}$ axons to that following 
183 application of a near-saturating concentration of exogenous dopamine (cf. Figure 1), we found

184 that the bulk endogenously released dopamine concentration was 5 times lower than near185 saturating concentrations of dopamine (Supplementary Figure 2D), although the effective 186 concentration at synaptic clefts may be higher.

187 We next tested whether we could visualize changes in cAMP levels in BA glutamatergic 188 neurons in response to photostimulated dopamine release (Figure 1G). We crossed DAT-Cre and 189 EMX1-Cre transgenic mice, which allowed for viral targeting of Chrimson to dopamine-releasing 190 neurons in the VTA and cADDis to glutamatergic neurons in the BA. Photostimulation of VTA ${ }^{\text {DA } \rightarrow B A}$ 191 axon terminals combined with two-photon imaging of cADDis revealed transient increases in 192 CAMP that peaked $\sim 5$ seconds later than dopamine, consistent with a delay between the rise in 193 extracellular dopamine and the generation of intracellular cAMP in target neurons (Supplementary 194 Figure 2E). The cAMP elevation remained above baseline for tens of seconds following optical 195 stimulation of VTA ${ }^{\mathrm{DA} \rightarrow \mathrm{BA}}$ axons - much longer than the duration of elevated extracellular dopamine 196 (Figure 1H). The slower decay of cAMP was not due to prolonged activation of target dopamine 197 receptors, as similar decay kinetics of evoked increases in cAMP were observed following direct 198 photostimulation of intracellular cAMP using a novel blue-light activated adenylate cyclase 199 (biPAC; Supplementary Figure 3A-C) (Zhang et al., 2021b). Thus, the release of dopamine onto 200 BA neurons is transformed into a transient elevation in intracellular cAMP that extends in time by 201 approximately five-fold compared to the elevation in extracellular dopamine (note that this is likely 202 an underestimate of the duration of cAMP elevations given the low affinity of cADDis for cAMP, $203 \mathrm{Kd} \sim 10 \mu \mathrm{M})$. This extended elevation in cAMP across tens of seconds may reflect an extended 204 window of plasticity that could result in generalization of plasticity to unpaired cues occurring 205 within that time window (e.g. Supplementary Figure S1).

We next examined endogenous dopamine-evoked cAMP signals from individual BA 208 neuron somata (Figure 1I,J; see Methods). Similar to cellular responses to exogenous dopamine 
application (Figure 1F), we detected widespread cAMP responses in over $70 \%$ of all BA

210 glutamatergic neurons as well as in the surrounding neuropil largely composed of dendrites of

211 these neurons (Figure $1 \mathrm{~K}, \mathrm{~L}$ ). While the magnitudes of the cAMP increases were small (Figure

$2121 \mathrm{M})$, they were larger than those observed following $1 \mu \mathrm{M}$ exogenous dopamine, a concentration

213 that also drove cAMP increases across all BA neurons (Figure 1F). We observed similar

214 magnitude responses in somatic and neuropil compartments, consistent with the finding that

215 dopaminergic synaptic terminals contact dendrites of BA glutamatergic neurons (Muller et al.,

216 2009) (Figure 1M). These findings indicate that most BA glutamatergic neurons express functional

217 D1 receptors and can respond to axonal release of dopamine.

Synaptic depression of dopamine release temporally restricts cAMP signals in BA neurons

We noticed that brief trains of optical stimulation $(20 \mathrm{~Hz} ; 2,5$, or $10 \mathrm{~s}$ duration) generated

221 transient elevations in cAMP that increased sublinearly with pulse train duration (Figure $2 \mathrm{~A}-\mathrm{C}$ ).

222 This sublinearity may reflect presynaptic depression of dopamine, as has been observed for

223 dopaminergic inputs to the striatum (Liu and Kaeser, 2019). Indeed, we observed that

224 photostimulating VTA ${ }^{\mathrm{DA} \rightarrow \mathrm{BA}}$ axons evoked dopamine release that scaled sublinearly with

225 increasing duration of the $15 \mathrm{~Hz}$ pulse train (Figure 2D-F). We also observed a diminished

226 magnitude of dopamine release when inter-trial intervals (ITIs) were 20 seconds long, but not

227 when they were two minutes long (Figure $2 \mathrm{G}-\mathrm{I}$ ). We found that 20 second ITIs resulted in a $25 \%$

228 depression in dopamine release whereas 120 second ITIs did not result in depression (Figure

$2292 \mathrm{H}, \mathrm{I})$. This long-lasting depression of dopamine release is consistent with the depression of

$230 V_{T A}{ }^{\mathrm{DA} \rightarrow \mathrm{BA}}$ axon-evoked glutamate co-released with dopamine in the BA (Lutas et al., 2019), and

231 with evidence that VTA projections to the striatum also exhibit synaptic depression when ITIs are

232 shorter than two minutes (Adrover et al., 2014). 

axons

We next asked whether cAMP dynamics in BA glutamatergic neurons exhibit similar

237 characteristics in vivo as in our brain slice experiments. We used fiber photometry to track

238 cADDis signals in BA glutamatergic neurons in response to photostimulation of Chrimson in

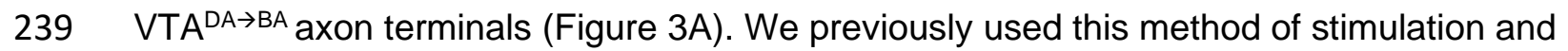

240 recording to measure optical stimulation-evoked dopamine release of a similar magnitude as

241 natural (shock-evoked) dopamine release (Lutas et al., 2019). We reliably detected cAMP

242 production in BA neurons in response to optically induced dopamine release in vivo (Figure 3B).

243 Similar to our slice measurements, we observed elevated cAMP levels lasting tens of seconds

244 following brief optical stimulation of dopamine release (Figure 3B). Our protocol for in vivo

245 photostimulation of VTA $\mathrm{DA}^{\mathrm{DA}}$ axons was well below saturating levels, as it drove weaker

246 changes in CAMP than intraperitoneal injection of a high concentration of a dopamine Type 1

247 receptor agonist (SKF81297; 20 mg/kg; Supplementary Figure 4A). When we repeatedly

248 photostimulated VTA ${ }^{\mathrm{DA} \rightarrow \mathrm{BA}}$ axons every $30 \mathrm{~s}$, we found that cAMP transients attenuated over

249 time, such that within three minutes, they were profoundly reduced in magnitude (Figure 3C,D).

250 This result led us to consider the possibility that the diminished responses were caused by

251 presynaptic depression of dopamine release, as described in Figure 2.

We explored this possibility by modeling cAMP dynamics in response to dopamine

254 released at variable ITIs (30 s or 10 s; Figure 3E; Supplementary Figure 4B). We started with the

255 assumption that the dynamics of intracellular cAMP in BA neurons following photostimulation of

256 dopamine axons could be understood as a simple convolution of the dynamics of evoked

257 dopamine concentration and of the decay in intracellular cAMP. We separately fit

258 monoexponential decay functions to the post-peak response time courses from slice recordings

259 for axon-evoked dopamine release and for cAMP evoked by direct, transient photostimulation of 
260 intracellular cAMP (dopamine: $\tau=4 \mathrm{~s}$; cAMP: $\tau=20 \mathrm{~s}$; see Methods). We then modeled the

261 expected exponential attenuation in dopamine release with repeated stimulation based on our

262 measurements of paired-pulse depression (Figure 2C; paired pulse ratio: 75\%). Indeed, our

263 simulation revealed that depression in dopamine release over trials is largely sufficient to result

264 in a return of evoked cAMP levels to baseline within three minutes (Figure 3E; Supplementary

265 Figure 4B).

266

We compared this simulation to in vivo fiber photometry of cADDis signals during the same

268 protocol of photostimulation of VTA ${ }^{\mathrm{DA} \rightarrow B A}$ axons with 30 - or 10-second ITIs. We found that

269 endogenous cAMP dynamics mirrored those in our simulation (Figure 3F; Supplementary Figure

$2704 \mathrm{C})$. To further assess whether a presynaptic rather than postsynaptic mechanism mediated the

271 depression in evoked cAMP responses, we used biPAC to bypass the dopamine receptor and

272 directly stimulate cAMP production (Figure 3G). When we photostimulated biPAC repeatedly

273 every 30 seconds, cAMP levels remained persistently elevated through the duration of the

274 recording (Figure $3 \mathrm{H})$. Together, these results demonstrate that transient cAMP elevations such

275 as those that likely occur during a rapid sequence of salient events do not accumulate across

276 minutes, likely because of presynaptic depression of dopamine release.

278 Repeated salient events evoke decreasing cAMP responses in BA neurons

279 Dopamine is naturally released in the BA during motivationally salient appetitive and 280 aversive events (Lutas et al., 2019). We tested whether we could detect cAMP changes during 281 unexpected delivery of appetitive palatable food in hungry mice or aversive unexpected tail 282 shocks in sated mice (Figure 4A), both of which drive robust dopamine release in the BA (Lutas 283 et al., 2019). On the first trial, we were able to detect significant changes in response to the 284 consumption of unexpected food delivery or to the delivery of an unexpected tail shock (Figure $2854 \mathrm{~B}, \mathrm{C})$, albeit with a lower amplitude than that observed during photostimulation. Similar to our 
286 finding with repeated photostimulation of VTA ${ }^{\mathrm{DA} \rightarrow \mathrm{BA}}$ axons, we found that repeated food delivery

287 or tail shock resulted in attenuated cAMP transients, such that within 5 minutes, we were unable

288 to detect significant evoked cAMP increases (Figure 4D,E). The depression of cAMP responses

289 in vivo in response to tail shocks or food delivery that was repeated every 30 or 10 seconds was

290 similar to expectations from our simulated dopamine-evoked cAMP signal (Figure 4F,G;

291 Supplementary Figure 4D,E).

292 Finally, we addressed whether depression of cAMP signals also resulted in depression

293 rather than accumulation of protein kinase $A(P K A)$ activity - a key downstream regulator of

294 synaptic plasticity (Figure 4H). We use fiber photometry and a fluorescent sensor of PKA activity,

295 exrai-AKAR2 (Zhang et al., 2021a), to detect in vivo responses to tail shocks (Figure $4 \mathrm{H}$ ). We

296 found that unexpected tail shock delivery elicited responses in the exrai-AKAR2 photometry

297 signals that were more pronounced than in our cADDis recordings (Figure 4I). We then tested if

298 repeated delivery of tail shocks every 30 s produced a depressing exrai-AKAR2 response. Indeed,

299 consistent with the depression in the evoked cAMP response magnitude, we found that exrai-

300 AKAR2 responses depressed with repeated unexpected tail shocks (Figure 4I). While exrai-

301 AKAR2 reports the ratio of phosphorylation by PKA and dephosphorylation by protein

302 phosphatases, our earlier findings of depressing cAMP responses to repeated dopamine axon

303 photostimulation suggests a weakening of cAMP-mediated PKA activation. However, we cannot

304 fully rule out the possibility that increases in protein phosphatase activity also contribute to the

305 depression in exrai-AKAR2 signal. Together, our characterization of ex vivo and in vivo cAMP

306 and PKA sensor responses following endogenous dopamine release reveals temporal constraints

307 on sustained cAMP elevation and PKA phosphorylation of targets in BA neurons. These temporal

308 constraints likely affect the potency of associative plasticity of novel vs. rapidly repeated salient

309 events.

\section{Discussion}


The release of neuromodulators including dopamine in basal amygdala is critical for cue-

313 outcome association learning (Johansen et al., 2014; Tang et al., 2020). Dopamine receptor

314 activation, via cAMP dynamics, may strengthen synaptic inputs to enhance the salience of

315 stimuli paired with either appetitive or aversive outcomes (Lutas et al., 2019). Here, we establish

316 an imaging approach to investigate this process in BA glutamatergic neurons using optical tools

317 to monitor cAMP dynamics and to evoke dopamine release or drive direct cAMP production. We

318 demonstrate that exogenous dopamine as well as dopamine release from VTA ${ }^{\mathrm{DA} \rightarrow \mathrm{BA}}$ axon

319 terminals can increase cAMP in all BA glutamatergic neurons. Given that BA glutamatergic

320 neurons segregate into appetitive- or aversive-preferring neurons, this widespread dopamine-

321 evoked cAMP signal ignores the boundaries imposed by valence-specific teaching signals.

We found that during stimulation protocols that mimic temporally clustered, salient

324 events, VTA ${ }^{\mathrm{DA} \rightarrow \mathrm{BA}}$ axons initially drive cAMP in BA neurons in vivo. However, synaptic

325 depression of these VTA ${ }^{\mathrm{DA} \rightarrow \mathrm{BA}}$ axons limits the temporal window of dopamine-related

326 accumulation of CAMP. In contrast, direct, repeated photostimulation of cAMP production that

327 bypasses the dopamine receptor does not show this depression, and instead drives a persistent

328 elevation in cAMP levels. These and other findings confirm that depression of dopaminergic

329 input and actions of phosphodiesterases (Supplementary Figure 3C) control the accumulation of

330 CAMP in BA neurons, thereby potentially regulating windows of plasticity in this region. More

331 generally, our findings regarding the dynamics of dopamine release and cAMP accumulation in

332 BA in vitro and in vivo provide a platform for linking the dynamics of intracellular biochemical

333 signals with the dynamics of within-trial, across-trial, and across-day plasticity and learning.

\section{Synaptic depression of dopamine release in BA restricts the window of elevated cAMP}

336 when release events are clustered in time. 
We found a potent and long-lasting depression of dopamine-evoked cAMP transients.

338 We showed that synaptic depression of dopamine release occurs at VTA ${ }^{\mathrm{DA} \rightarrow \mathrm{BA}}$ terminals and

339 that modeling this synaptic depression fully captures the weakened cAMP signals we recorded

340 in vivo in response to photostimulated dopamine release. While to our knowledge this property

341 had not been measured previously at the VTA ${ }^{\mathrm{DA} \rightarrow \mathrm{BA}}$ synapse, similar characteristics have been

342 observed in dopamine neuron projections to the dorsal and ventral striatum (Adrover et al.,

343 2014). Synaptic depression at these mesostriatal dopaminergic synapses is mediated by

344 several factors including the activation of presynaptic Type 2 dopamine receptors following

345 release of dopamine, and by depletion of the synaptic vesicle pool (Liu and Kaeser, 2019). We

346 suspect that similar mechanisms mediate the minutes-long depression we observed in

347 VTA ${ }^{\mathrm{DA} \rightarrow \mathrm{BA}}$ projections, both in slices and in vivo. We demonstrated that this depression greatly

348 impairs the ability of dopamine axon activation to maintain elevated postsynaptic cAMP levels.

349 Thus, depression of dopamine release may impose temporal constraints on synaptic plasticity

350 when salient events are clustered in time. It will be interesting to test whether clustering together

351 or spacing apart salient events using the temporal information obtained from cAMP

352 measurements influences learning rates or memory consolidation in amygdala-dependent tasks,

353 as has been observed in Drosophila (Jacob and Waddell, 2020). Moreover, as certain

354 medications (e.g. methylphenidate) as well as drugs of abuse (e.g. cocaine) can enhance

355 dopamine synaptic depression (Adrover et al., 2014), future studies can investigate how these

356 drugs influence the temporal properties of amygdala plasticity.

358 Is a permissive plasticity signal from VTA ${ }^{\mathrm{DA} \rightarrow \mathrm{BA}}$ axons broadcast widely or only to a

359 subset of BA neurons?

360 A major goal of our efforts to image cAMP in BA neurons was to understand whether

361 dopamine, which is released from individual VTA ${ }^{\mathrm{DA} \rightarrow \mathrm{BA}}$ axons following both appetitive and

362 aversive outcomes (Lutas et al., 2019), drives increases in cAMP in all BA glutamatergic 
neurons. This could allow for dopamine to encode the motivational salience of an event by scaling the proportion of BA neurons that undergo a rise in cAMP levels. If different BA neurons exhibit varying sensitivities to dopamine, greater dopamine release during events with higher motivational salience should result in a greater percentage of BA neurons with dopamineevoked changes in downstream signaling. We found that exogenous dopamine could elevate cAMP in all BA glutamatergic neurons, and photostimulation of endogenous release from dopamine axons drove detectable cAMP responses in most BA glutamatergic neurons in slices.

370 On the one hand, this is likely to be a conservative estimate of affected neurons, given the

371 limited sensitivity of our cAMP sensor (see below). On the other hand, we found that

372 endogenous release of dopamine in response to palatable food and unexpected tail shocks

373 were weaker than the photostimulated release, consistent with the interpretation that fewer BA

374 neurons may have elevated cAMP following natural release of dopamine. The depression in

375 dopamine-evoked cAMP we observed could further limit the magnitude of cAMP in some BA

376 neurons. Thus, if different BA neurons have varying magnitudes of dopamine-evoked cAMP, the

377 size of the BA neuronal population that exhibits plasticity could be limited to those neurons with

378 the largest cAMP response. These results suggest that dopamine may determine the

379 percentage of BA neurons that are plastic at a given time, thereby scaling learning rates with the 380 motivational salience of expected appetitive and aversive outcomes.

Potential limitations imposed by the sensitivity of the cAMP sensor.

The affinity of the cAMP sensor we used is in the low micromolar range (Tewson et al.,

384 2016), which means that changes in cAMP concentration in the nanomolar range would be

385 outside of this sensitivity range. Especially in the case of our in vivo fiber photometry

386 measurements, we may have missed changes in cAMP in response to unexpected delivery of

387 palatable food or tail shocks. We overcame this limitation via additional experiments using a

388 fluorescent biosensor of PKA activity (Zhang et al., 2021a), which had improved signal-to-noise 
in response to unexpected tail shocks and revealed clear depression with repeated salient events. An additional limitation is that our sensor measurements were not targeted specifically

391 to dendritic compartments where cAMP increases may be concentrated, and thus our bulk

392 measurements across somatic and dendritic compartments may have limited our sensitivity

393 further. Nevertheless, presynaptic depression of dopamine release likely limits cAMP and PKA

394 signals both in dendritic and somatic compartments. Future studies using higher affinity cAMP

395 sensors (Klarenbeek et al., 2015) as well as sensors targeted to dendritic spines (e.g. by fusing

396 with PSD-95) should allow for further real-time interrogation of these signals in response to

397 endogenous salient events.

\section{Conclusions and future directions.}

We have established an imaging platform using reporters and actuators of intracellular

cAMP that allowed for direct interrogation of dopamine-dependent cAMP signals in BA

402 glutamatergic neurons in vitro and in vivo. Future studies can employ this approach to

403 investigate cAMP dynamics in BA neurons in pre-clinical settings, such as following acute and

404 chronic exposure to addictive substances, or to stressful, traumatic events. In addition, many

405 other critical neuromodulatory signals in the BA (e.g., norepinephrine, PACAP, and serotonin)

406 act via influences on cAMP. A similar imaging approach could be used to investigate how these

407 additional neuromodulators affect cAMP levels alone and in conjunction with dopamine

408 signaling. Another key future direction will involve examination of cAMP dynamics in non-

409 glutamatergic targets of VTA dopamine neurons in BA, such as parvalbumin-positive

410 interneurons (Chu et al., 2012; Pinard et al., 2008). In conclusion, we have revealed important

411 temporal and spatial characteristics of dopamine actions via cAMP signaling in BA, which have

412 informed hypotheses about the role of dopamine during associative learning. Importantly, while

413 most in vivo studies of cellular plasticity have focused on changes in electrical and calcium 
414 activity, continued optimization of tools to detect molecular signaling cascades will expand our

415 understanding of the underlying biochemical signals that control neural circuit plasticity.

417 Acknowledgements

418 We thank members of the Andermann lab including K. Evans, R. Essner, N. Nguyen, K. McGuire,

419 Dr. H. Kucukdereli, Dr. O. Amsalem, Dr. J.S. Alvarado for helpful feedback on the manuscript.

420 We thank V. Flores-Maldonado for assistance with mouse colony care. We thank Dr. J. Madara

421 for assistance with brain slice preparation. Boston Children's Hospital Viral Core provided viral

422 packaging services. We thank Drs. Ingie Hong and Richard Huganir for sharing exrai-AKAR2

423 virus. Authors were supported by an NIH F32 DK112589, a Davis Family Foundation Award, and

424 a Boston Nutrition Obesity Research Center Pilot grant (A.L.), a Lefler Fellowship (S.X.Z.), NIH

425 R01 DK109930, DP1 AT010971, DP1 AT010971-02S1, R01 MH12343, the McKnight

426 Foundation, the Klarman Family Foundation, and the Harvard Brain Science Initiative Bipolar

427 Disorder Seed Grant, supported by Kent and Liz Dauten (M.L.A).

428 The authors declare no conflicts of interest.

430 Author contributions

431 A.L. and M.L.A. conceived the project and wrote the manuscript. A.L. designed and performed

432 brain slice and two-photon imaging. A.L., K.F. and A.S. conducted fiber photometry recordings.

433 A.L., K.F., A.S. and S.X.Z. performed surgical procedures. A.L. and S.X.Z. designed experiments 434 for photostimulation concurrent with two-photon imaging of acute brain slices. A.L. analyzed all 435 data. 
440 A) Top: DNA construct for human Synapsin (hSyn) promotor driven, Cre-dependent expression

441 of cADDis in neurons. Black and green triangles: Cre recombination sites. WPRE: woodchuck

442 hepatis virus post-transcriptional regulatory element. Bottom: viral injection strategy for selective

443 expression of cADDis in glutamatergic neurons of the basal amygdala (BA) using Emx1-Cre

444 transgenic mice.

445 B) Left: schematic of live cell epifluorescence imaging of cADDis in a brain slice. Right:

446 representative image of amygdala brain slice expressing cADDis in BA neurons. CeA: central

447 amygdala. LA: lateral amygdala.

448 C) Left: forskolin-mediated activation of adenylate cyclase (AC) drives production of cAMP.

449 Middle: example forskolin-evoked $(50 \mu \mathrm{M})$ cADDis fluorescence change in BA region of a brain

450 slice. Y-axis is inverted for clarity, as increases in cAMP decreased cADDis fluorescence. Right:

451 peak response of individual slices. Mean \pm s.e.m. $n=5$ slices from 2 mice.

452 D) Left: dopamine-mediated activation or suppression of adenylate cyclase (AC) via activation

453 of either Type 1 (D1R) or Type 2 (D2R) dopamine receptors. Middle: example dopamine-evoked

$454(20 \mu \mathrm{M})$ cADDis fluorescence change in BA. Right: peak response of individual slices. Mean \pm

455 s.e.m. $\mathrm{n}=4$ from 2 mice.

456 E) Left: epifluorescence image of BA glutamatergic neurons expressing cADDis. Right: masks

457 for individual cell somata.

458 F) Average cumulative distribution of peak responses of individual BA neurons to dopamine and

459 forskolin application. $1 \mu \mathrm{M}$ dopamine: $\mathrm{n}=440$ neurons from 3 slices from 1 mouse. $3 \mu \mathrm{M}$

460 dopamine: $n=294$ neurons from 2 slices from 2 mice. $20 \mu \mathrm{M}$ dopamine: $\mathrm{n}=579$ neurons from 4

461 slices from 2 mice. $50 \mu \mathrm{M}$ forskolin: $\mathrm{n}=597$ neurons from 5 slices from 2 mice. ${ }^{\text {n.s. }}, \mathrm{p}>0.05$.

462 Kolmogorov-Smirnov test. 
G) Schematic of brain slice imaging of cADDis fluorescence and photostimulation-evoked

464 dopamine release. VTA ${ }^{\mathrm{DA} \rightarrow \mathrm{BA}}$ dopamine axons expressing Chrimson were stimulated using red-

465 light illumination from below the slice. cAMP levels were determined by two-photon imaging of

466 cADDis in BA glutamatergic neurons.

467 H) Mean cADDis fluorescence in response to photostimulation of dopamine release (10 s

468 duration; $15.5 \mathrm{~Hz}$ ). Mean \pm s.e.m. $\mathrm{n}=12$ slices from 4 mice. Inset: mean cADDis fluorescence

469 with monoexponential fit overlaid (blue line). Fitted decay rate was $20 \mathrm{~s}$.

470 I) Left: maximum intensity projection of a volume (15 depths, $\sim 10 \mu \mathrm{m}$ apart) of cADDis

471 fluorescence. The image color is inverted for clarity. Right: maximum projection of the color-

472 coded cell masks of individual BA neurons from the imaged volume.

$473 \mathrm{~J})$ Image of normalized difference in cADDis fluorescence between dopamine and baseline

474 conditions in an example slice field of view.

475 K) Cumulative distribution of peak cADDis responses of individual BA neurons following

476 photostimulation of VTA ${ }^{\mathrm{DA} \rightarrow \mathrm{BA}}$ axon terminals. $\mathrm{n}=1697$ neurons from 11 slices from 5 mice.

477 L) Percentage of all neuronal somata (black) or neuropil rings surrounding somata (blue) per

478 slice with significant cADDis responses following photostimulation of VTA ${ }^{\mathrm{DA} \rightarrow \mathrm{BA}}$ axons. Mean \pm

479 s.e.m. $\mathrm{n}=11$ slices from 5 mice.

480 M) Peak response of neuronal somata or neuropil rings with significant cADDis responses

481 following photostimulation of VTA ${ }^{\mathrm{DA} \rightarrow \mathrm{BA}}$ axons. Mean \pm s.e.m. $\mathrm{n}=11$ slices from 5 mice.

483 Figure 2. Synaptic depression of dopamine release restricts postsynaptic cAMP

484 accumulation

485 A) Schematic of brain slice imaging of cADDis fluorescence and photostimulation-evoked 486 dopamine release. VTA ${ }^{\mathrm{DA} \rightarrow \mathrm{BA}}$ axons expressing Chrimson were stimulated using red-light

487 illumination from below the slice. cAMP levels were determined by two-photon imaging of 488 cADDis expressed in BA glutamatergic neurons. 
B) Left: example recording of cADDis fluorescence in response to a train of red-light pulses (10

490 s duration; $15.5 \mathrm{~Hz}$ ). Right: peak response of individual brain slices to photostimulation of

491 dopamine release for $2 \mathrm{~s}(\mathrm{n}=7$ slices from 4 mice), $5 \mathrm{~s}(\mathrm{n}=11$ slices from 5 mice $)$, or $10 \mathrm{~s}(\mathrm{n}=$

49213 slices from 5 mice). Mean \pm s.e.m.

493 C) Schematic of brain slice imaging of photostimulation-evoked dopamine release. Dopamine

494 levels were determined by two-photon imaging of dLight1.1 expressed in BA.

495 D) Left: example recording of dLight1.1 fluorescence in response to a train of red-light pulses

496 (10 s duration; $15.5 \mathrm{~Hz} ; 620 \mathrm{~nm}$ ). Right: peak responses of average signal from individual brain

497 slices to photostimulation of dopamine release for $2 \mathrm{~s}(\mathrm{n}=13$ slices from 4 mice), $5 \mathrm{~s}(\mathrm{n}=12$

498 slices from 4 mice), or $10 \mathrm{~s}(\mathrm{n}=16$ slices from 4 mice). Mean \pm s.e.m.

499 E) Left: mean time course in response to a pair of photostimulation trials (2 s pulse train

500 duration; $15.5 \mathrm{~Hz} ; 20 \mathrm{~s} \mathrm{ITI}$ ) normalized to first peak. Mean \pm s.e.m. $\mathrm{n}=13$ slices from 4 mice.

501 Right: same as left but for 120 s ITI. $\mathrm{n}=7$ slices from 2 mice.

502 F) Paired pulse ratio of peak response (second pulse train / first pulse train) for inter-trial

503 intervals (ITIs) of $20 \mathrm{~s}\left(^{* * *}, p=0.0005, n=13\right.$ slices from 4 mice) or $120 \mathrm{~s}$ (n.s., $p=0.57, n=7$

504 slices from 2 mice) duration. Two-sided Wilcoxon sign-rank. Mean \pm s.e.m.

505

506 Figure 3. cAMP signals track photostimulated dopamine release in vivo

507 A) Schematic of in vivo photometry recordings of cADDis fluorescence and photostimulation of

508 dopamine release. VTA ${ }^{\mathrm{DA} \rightarrow \mathrm{BA}}$ dopamine axons expressing Chrimson were stimulated using red-

509 light illumination via the same implanted fiber used to record cADDis signals from BA

510 glutamatergic neurons.

511 B) Mean time course of $c A D D i s$ fluorescence in response to photostimulation of VTA ${ }^{D A} \rightarrow B A$

512 axons (2 s duration; $20 \mathrm{~Hz}$ ). $\mathrm{n}=5$ mice. Mean \pm s.e.m. 
513 C) Mean time course of $C A D D$ is fluorescence of response to photostimulation of VTA ${ }^{D A} \rightarrow B A$

514 axons $(2 \mathrm{~s}$ duration; $20 \mathrm{~Hz}$ ) for first trial of recording (left) or fourth minute of recording (right:

515 average of 2 trials of $30 \mathrm{~s}$ duration). $n=5$ mice. Mean \pm s.e.m.

516 D) Peak response following photostimulation on first trial vs. fourth minute $\left({ }^{*}, p=03, n=5\right.$

517 mice). Two-tailed paired t-test. Mean \pm s.e.m.

518 E) Left: simulation of dopamine (top; red) and cAMP (bottom; blue) dynamics for a single 2 s-

519 duration activation. Right: simulation of stimulation of dopamine release at $30 \mathrm{~s}$ intervals.

520 F) Mean time course of cADDis fluorescence (black line) of response to photostimulation of

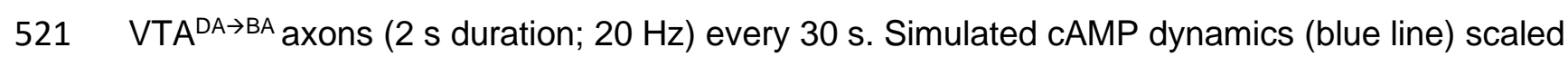

522 to peak photometry amplitude. Mean \pm s.e.m. $n=5$ mice.

523 G) Schematic of cAMP production using biPAC (blue-light stimulation of adenylate cyclase).

524 H) Direct stimulation of cAMP production by blue-light activation of biPAC in brain slices (black;

5252 s duration; $30 \mathrm{~s} \mathrm{ITI).} \mathrm{Simulated} \mathrm{cAMP} \mathrm{dynamics} \mathrm{(blue} \mathrm{line)} \mathrm{scaled} \mathrm{to} \mathrm{peak} \mathrm{photometry}$

526 amplitude. Mean \pm s.e.m. $n=11$ slices from 4 mice.

527

528 Figure 4. In vivo fiber photometry recordings of cAMP dynamics follow tail shock or

529 Ensure delivery

530 A) Left: schematic of delivery of unexpected aversive tail shock or palatable food (Ensure) to an

531 awake, head-fixed mouse. Middle: predicted consequences of tail shock or Ensure delivery on

532 cAMP production in BA glutamatergic neurons. Right: schematic of in vivo photometry

533 recordings of cADDis fluorescence.

534 B) Left: mean time course of cADDis response to unexpected tail shock delivery $(0.3 \mathrm{~mA} ; 50 \mathrm{~ms}$

535 duration) or unexpected Ensure delivery via a solenoid (single $15 \mu \mathrm{L}$ droplet) during first trial.

536 Right: same as left, but for the fourth minute of recording. $\mathrm{n}=5$ mice. Mean \pm s.e.m. 
537 C) Peak response following tail shock or Ensure delivery during the first trial (left) or fourth 538 minute (right). ${ }^{* * *}, \mathrm{p}=0.0004 ;{ }^{*}, \mathrm{p}=0.03$, ${ }^{\text {n.s. }}, \mathrm{p}>0.05, \mathrm{n}=5$ mice. Two-tailed t-test. Mean \pm 539 s.e.m.

540 D) Left: mean time course of cADDis fluorescence in response to unexpected tail shock delivery

541 (0.3 mA; 50 ms duration) every $30 \mathrm{~s}$. Right: mean time course of cADDis fluorescence in

542 response to unexpected Ensure delivery (single $15 \mu \mathrm{L}$ droplet) every $30 \mathrm{~s}$. Recording

543 normalized to baseline period before first trial. Mean \pm s.e.m. $n=5$ mice. Simulated cAMP

544 dynamics (blue line; based on model shown in Figure 3E) scaled to peak photometry amplitude.

545 E) Left: predicted consequences of tail shock on protein kinase A (PKA) activity.

546 Dephosphorylation by protein phosphatases (PP) also indicated. Right: schematic of in vivo

547 photometry recordings of fluorescence intensity of exrai-AKAR2 (biosensor of PKA activity, as

548 measured by phosphorylation of PKA substrate peptide).

549 F) Mean time course of exrai-AKAR2 fluorescence in response to unexpected tail shock delivery

550 (0.3 mA; $50 \mathrm{~ms}$ duration) every $30 \mathrm{~s}$. Recording normalized to baseline period before first trial.

551 Mean \pm s.e.m. $\mathrm{n}=6$ mice. Simulated cAMP dynamics (blue line; based on model shown in

552 Figure 3E) scaled to peak photometry amplitude.

554 Supplementary Figure 1. Two-photon calcium imaging during association of visual 555 stimulus and photostimulation of VTA ${ }^{\mathrm{DA} \rightarrow B A}$ axons

556 A) Left: schematic of in vivo two-photon imaging of GCaMP6s fluorescence and

557 photostimulation of dopamine release. VTA ${ }^{\mathrm{DA} \rightarrow \mathrm{BA}}$ dopamine axons expressing Chrimson were 558 stimulated using red-light illumination via the same implanted lens used to record GCaMP6s 559 signals from BA glutamatergic neurons. Right: schematic of two-photon microscope optical path 560 for red-light illumination and concurrent collection of green GCaMP6s fluorescence emission.

561 B) Top: example image of GCaMP6s expression in BA glutamatergic neurons. Bottom: image of 562 VTA ${ }^{\mathrm{DA} \rightarrow \mathrm{BA}}$ axons expressing Chrimson-tdTomato in the same field of view. 
563 C) Diagram of trial structure design for pairing one visual stimulus (vertically oriented drifting

564 bars, "Cue A"; 2 s duration) followed 200 ms later by photostimulation ( 5 mW, 2 s duration; 15.5

$565 \mathrm{~Hz}$ ). A second visual stimulus (horizontally oriented drifting bars, "Cue B"; 2 s duration) was not

566 directly paired with photostimulation.

567 D) Percentage of all neurons with significant cue responses on "Day 0" before photostimulation

568 (Cue A: 14/1283 neurons; Cue B: 14/1283 neurons from 7 mice) and following three days of

569 photostimulation (Cue A: 48/1199 neurons; Cue B: 12/1199 neurons from 6 mice).

570 E) Heatmap with rows depicting mean response of BA neurons ( $n=48$ neurons, 14 fields of

571 view from 6 mice) during presentation of each cue on the third day of photostimulation.

572 F) Mean time course of responses to visual stimuli and photostimulation across all BA neurons

573 that were significantly activated (red; $n=18$ neurons) or suppressed (blue; $n=30$ neurons) by

574 "Cue A".

575 G) Mean response to "Cue A" or "Cue B" for activated (left, n = 18 neurons from 6 mice, ${ }^{* * *}, p<$

5760.001 ) and suppressed neurons (right, $\mathrm{n}=30$ neurons from 6 mice, ${ }^{* * *}, \mathrm{p}<0.001$ ). Error bars:

577 s.e.m. across neurons. Two-sided Wilcoxon sign-rank.

578 H) Percentage of cue-responsive neurons preferring (i.e., maximally responsive to) a given cue

579 following three days of photostimulation (Cue A: 48/60 neurons; Cue B: 12/60 neurons).

581 Supplementary Figure 2. Photostimulation of VTA ${ }^{\mathrm{DA} \rightarrow B A}$ axons transiently elevates 582 dopamine in BA.

583 A) Schematic of brain slice imaging of photostimulation-evoked dopamine release. VTA ${ }^{\mathrm{DA} \rightarrow B A}$

584 dopamine axons expressing Chrimson were stimulated using red-light illumination from below

585 the slice. Dopamine levels were determined by two-photon imaging of dLight1.1 expressed in

586 BLA neurons.

587 B) Mean dLight1.1 fluorescence during photostimulation of dopamine release (5 s duration; 15.5

$588 \mathrm{~Hz}) . \mathrm{n}=14$ slices from 5 mice. Mean \pm s.e.m. 
C) Application of dopamine D1 receptor antagonist (SCH23390; $300 \mathrm{nM})$ blocks photostimulation-evoked dLight1.1 signal. Right: peak photostimulation-evoked dLight1.1

591 response before and after antagonist. ${ }^{* *}, p=0.006, n=7$ slices from 2 mice. Two-tailed paired 592 t-test. Mean \pm s.e.m.

593 D) Left: mean time course in response to photostimulation (5 s duration; $15.5 \mathrm{~Hz}$ ) followed by 594 application of dopamine $(3 \mu \mathrm{M})$. Mean \pm s.e.m. Right: response ratio of exogenous dopamine 595 vs. photostimulation-evoked dopamine release. $n=14$ slices from 5 mice. Mean \pm s.e.m.

596 E) Top: mean time course of dLight1.1 fluorescence change in response to photostimulation of 597 VTA ${ }^{\mathrm{DA} \rightarrow \mathrm{BA}}$ axons for 2, 5, or $10 \mathrm{~s}$. Bottom: mean time course of cADDis fluorescence changes in 598 response to photostimulation of VTA ${ }^{\mathrm{DA} \rightarrow \mathrm{BA}}$ axons for 2,5 , or $10 \mathrm{~s}$. elevated cAMP

A) Schematic of cAMP production using biPAC (blue-light stimulation of adenylate cyclase).

B) Example recording of cADDis fluorescence during biPAC photostimulation (2 s duration;

604 continuous illumination). Recording performed at $32^{\circ} \mathrm{C}$.

605 C) Stimulation of biPAC (2 s duration) in control slices (black; $n=13$ slices from 4 mice) or in 606 the presence of a phosphodiesterase inhibitor (IBMX, $100 \mu \mathrm{M}$; red; $\mathrm{n}=4$ slices from 4 mice). 607 Recording performed at room temperature. Mean \pm s.e.m.

\section{Supplementary Figure 4. Additional fiber photometry of cADDis}

610 A) Left: mean time course of cADDis fluorescence in response to intraperitoneal injection of a

611 Type 1 dopamine receptor (D1) agonist (blue; SKF81297, 20 mg/kg, $150 \mu \mathrm{L} ; \mathrm{n}=6 \mathrm{mice}$ ) or

612 saline (gray, $150 \mu \mathrm{L} ; \mathrm{n}=5$ mice). Right: response ratio of injected D1 agonist to photostimulated 613 dopamine response. $\mathrm{n}=6$ mice. Mean \pm s.e.m. 
614 B) Simulation of repeated $2 \mathrm{~s}$-duration stimulations of dopamine release occurring with $10 \mathrm{~s}$

615 inter-trial intervals (ITI).

616 C) Mean time course of cADDis fluorescence (black line) of response to photostimulation of

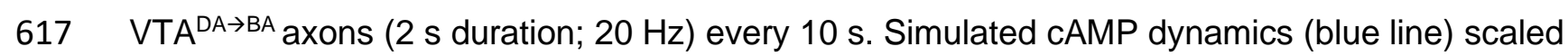

618 to peak photometry amplitude. Mean \pm s.e.m. $n=5$ mice.

619 D) Mean time course of cADDis fluorescence in response to unexpected tail shock delivery $(0.3$

$620 \mathrm{~mA} ; 50 \mathrm{~ms}$ duration) delivered every 10 s. Mean \pm s.e.m. $n=4$ mice. Simulated cAMP dynamics

621 (blue line) scaled to peak photometry amplitude.

622 E) Mean time course of cADDis fluorescence in response to unexpected Ensure delivery (single

$62315 \mu \mathrm{L}$ droplet) delivered every $10 \mathrm{~s}$. Simulated cAMP dynamics (blue line) scaled to peak

624 photometry amplitude. Mean \pm s.e.m. $n=3$ mice. 
626 Adrover, M.F., Shin, J.H., and Alvarez, V.A. (2014). Glutamate and dopamine transmission from

627 midbrain dopamine neurons share similar release properties but are differentially affected by

628 cocaine. J. Neurosci. 34, 3183-3192.

629 Bäckman, C.M., Malik, N., Zhang, Y., Shan, L., Grinberg, A., Hoffer, B.J., Westphal, H., and

630 Tomac, A.C. (2006). Characterization of a mouse strain expressing Cre recombinase from the 3'

631 untranslated region of the dopamine transporter locus. Genesis 44, 383-390.

632 Bissière, S., Humeau, Y., and Lüthi, A. (2003). Dopamine gates LTP induction in lateral

633 amygdala by suppressing feedforward inhibition. Nat. Neurosci. 6, 587-592.

634 Chu, H.-Y., Ito, W., Li, J., and Morozov, A. (2012). Target-specific suppression of GABA release

635 from parvalbumin interneurons in the basolateral amygdala by dopamine. J. Neurosci. 32,

636 14815-14820.

637 Correia, S.S., and Goosens, K.A. (2016). Input-specific contributions to valence processing in

638 the amygdala. Learn. Mem. 23, 534-543.

639 Handler, A., Graham, T.G.W., Cohn, R., Morantte, I., Siliciano, A.F., Zeng, J., Li, Y., and Ruta,

640 V. (2019). Distinct Dopamine Receptor Pathways Underlie the Temporal Sensitivity of

641 Associative Learning. Cell 178, 60-75.e19.

642 Jacob, P.F., and Waddell, S. (2020). Spaced Training Forms Complementary Long-Term

643 Memories of Opposite Valence in Drosophila. Neuron 106, 977-991.e4.

644 Johansen, J.P., Diaz-Mataix, L., Hamanaka, H., Ozawa, T., Ycu, E., Koivumaa, J., Kumar, A.,

645 Hou, M., Deisseroth, K., Boyden, E.S., et al. (2014). Hebbian and neuromodulatory mechanisms

646 interact to trigger associative memory formation. Proc. Natl. Acad. Sci. U. S. A. 111, E5584-

647 E5592.

648 Klapoetke, N.C., Murata, Y., Kim, S.S., Pulver, S.R., Birdsey-Benson, A., Cho, Y.K., Morimoto,

649 T.K., Chuong, A.S., Carpenter, E.J., Tian, Z., et al. (2014). Independent optical excitation of

650 distinct neural populations. Nat. Methods 11, 338-346. 
651 Klarenbeek, J., Goedhart, J., Van Batenburg, A., Groenewald, D., and Jalink, K. (2015). Fourth-

652 generation Epac-based FRET sensors for cAMP feature exceptional brightness, photostability

653 and dynamic range: Characterization of dedicated sensors for FLIM, for ratiometry and with high

654 affinity. PLoS One 10.

655 Liu, C., and Kaeser, P.S. (2019). Mechanisms and regulation of dopamine release. Curr. Opin.

656 Neurobiol. 57, 46-53.

657 Lutas, A., Kucukdereli, H., Alturkistani, O., Carty, C., Sugden, A.U., Fernando, K., Diaz, V.,

658 Flores-Maldonado, V., and Andermann, M.L. (2019). State-specific gating of salient cues by

659 midbrain dopaminergic input to basal amygdala. Nat. Neurosci. 22, 1820-1833.

660 Muller, J.F., Mascagni, F., and McDonald, A.J. (2009). Dopaminergic innervation of pyramidal

661 cells in the rat basolateral amygdala. Brain Struct. Funct. 213, 275-288.

662 Namburi, P., Beyeler, A., Yorozu, S., Calhoon, G.G., Halbert, S.A., Wichmann, R., Holden, S.S.,

663 Mertens, K.L., Anahtar, M., Felix-Ortiz, A.C., et al. (2015). A circuit mechanism for differentiating

664 positive and negative associations. Nature 520, 675-678.

665 O’leary, T.P., Sullivan, K.E., Wang, L., Clements, J., Lemire, A.L., and Cembrowski, M.S.

666 (2020). Extensive and spatially variable withincell-type heterogeneity across the basolateral

667 amygdala. Elife 9, 1-27.

668 O’Neill, P.-K., Gore, F., and Salzman, C.D. (2018). Basolateral amygdala circuitry in positive

669 and negative valence. Curr. Opin. Neurobiol. 49, 175-183.

670 Patriarchi, T., Cho, J.R., Merten, K., Howe, M.W., Marley, A., Xiong, W.-H., Folk, R.W.,

671 Broussard, G.J., Liang, R., Jang, M.J., et al. (2018). Ultrafast neuronal imaging of dopamine

672 dynamics with designed genetically encoded sensors. Science (80-. ). 360, eaat4422.

673 Pinard, C.R., Muller, J.F., Mascagni, F., and McDonald, A.J. (2008). Dopaminergic innervation

674 of interneurons in the rat basolateral amygdala. Neuroscience $157,850-863$.

675 Schoenbaum, G., Chiba, A.A., and Gallagher, M. (1999). Neural encoding in orbitofrontal cortex

676 and basolateral amygdala during olfactory discrimination learning. J. Neurosci. 19, 1876-1884. 
677 Steinberg, E.E., Keiflin, R., Boivin, J.R., Witten, I.B., Deisseroth, K., and Janak, P.H. (2013). A

678 causal link between prediction errors, dopamine neurons and learning. Nat. Neurosci. 16, 966-

679973.

680 Tang, W., Kochubey, O., Kintscher, M., and Schneggenburger, R. (2020). A VTA to basal

681 amygdala dopamine projection contributes to signal salient somatosensory events during fear

682 learning. J. Neurosci. 40, 3969-3980.

683 Tewson, P.H., Martinka, S., Shaner, N.C., Hughes, T.E., and Quinn, A.M. (2016). New DAG and

684 cAMP Sensors Optimized for Live-Cell Assays in Automated Laboratories. J. Biomol. Screen.

$68521,298-305$.

686 Tronson, N.C., Wiseman, S.L., Olausson, P., and Taylor, J.R. (2006). Bidirectional behavioral

687 plasticity of memory reconsolidation depends on amygdalar protein kinase A. Nat. Neurosci. 9,

688 167-169.

689 Yagishita, S., Hayashi-Takagi, A., Ellis-Davies, G.C.R., Urakubo, H., Ishii, S., and Kasai, H.

690 (2014). A critical time window for dopamine actions on the structural plasticity of dendritic

691 spines. Science (80-. ). 345, 1616-1620.

692 Zhang, X., and Li, B. (2018). Population coding of valence in the basolateral amygdala. Nat.

693 Commun. 9, 5195.

694 Zhang, J.F., Liu, B., Hong, I., Mo, A., Roth, R.H., Tenner, B., Lin, W., Zhang, J.Z., Molina, R.S.,

695 Drobizhev, M., et al. (2021a). An ultrasensitive biosensor for high-resolution kinase activity

696 imaging in awake mice. Nat. Chem. Biol. 17, 39-46.

697 Zhang, S.X., Lutas, A., Yang, S., Diaz, A., Fluhr, H., Nagel, G., Gao, S., and Andermann, M.L.

698 (2021b). Hypothalamic dopamine neurons motivate mating through persistent cAMP signalling.

699 Nat. $20211-5$.

700 
Figure 1

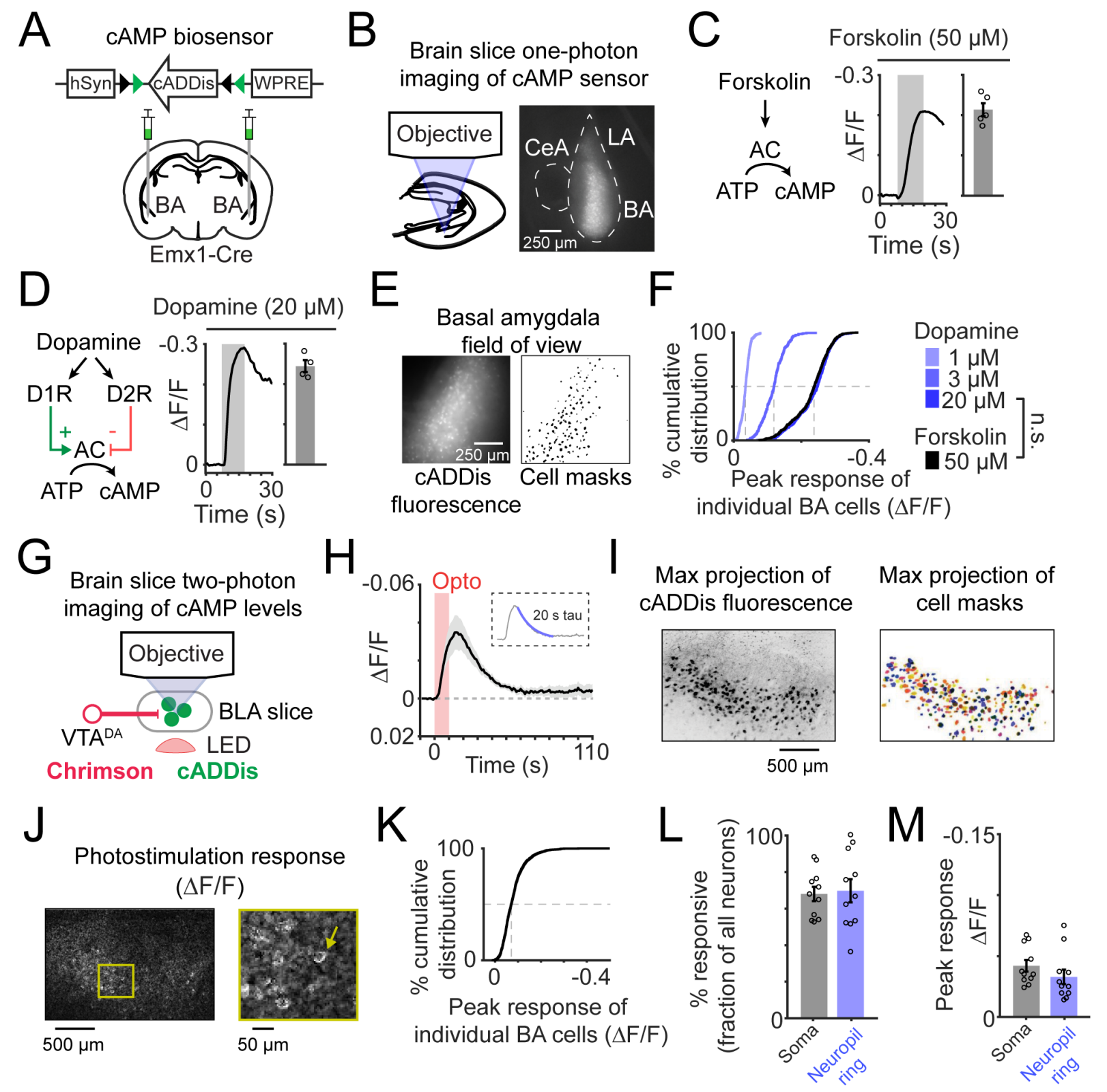




\section{Figure 2}
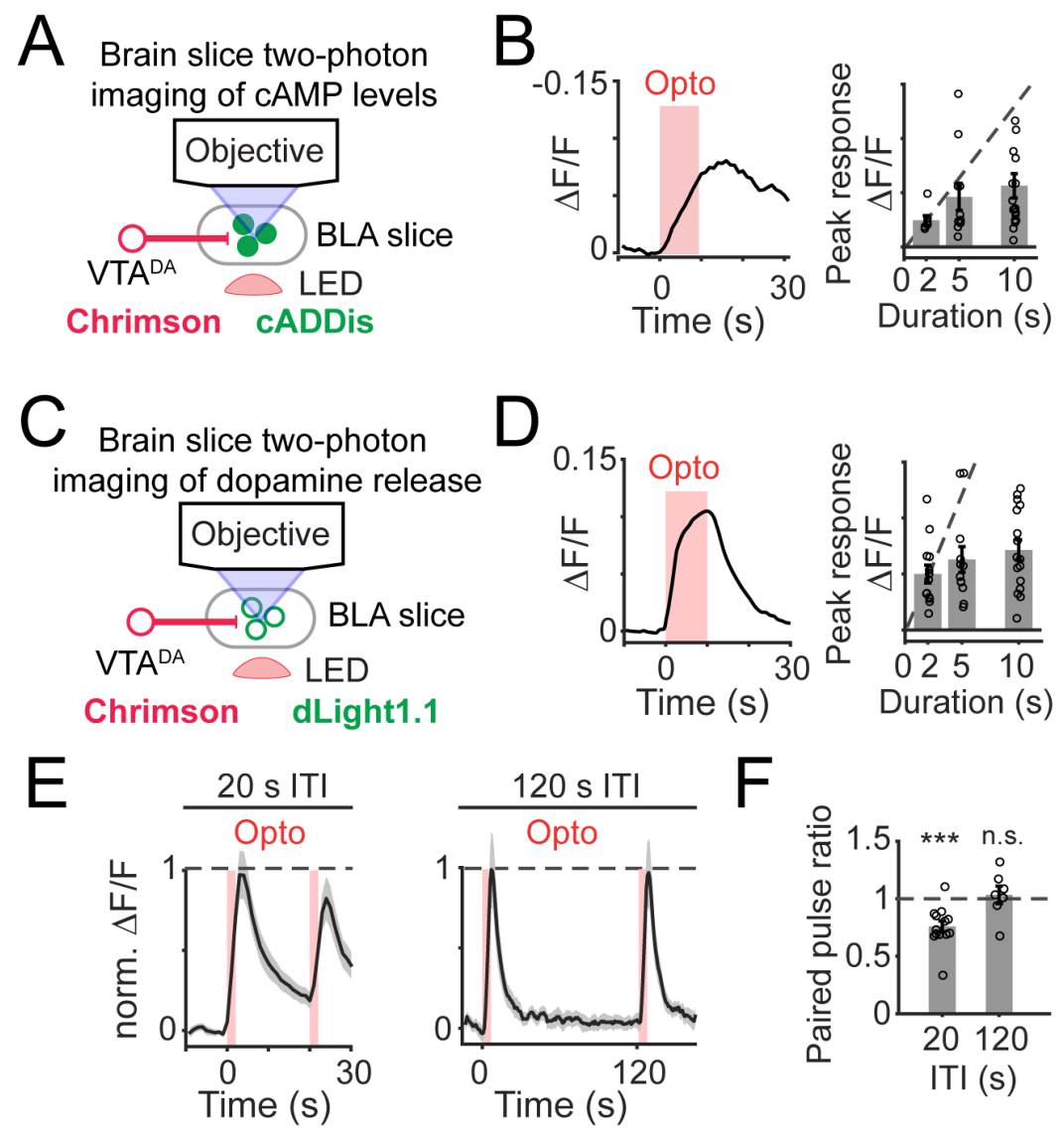


\section{Figure 3}

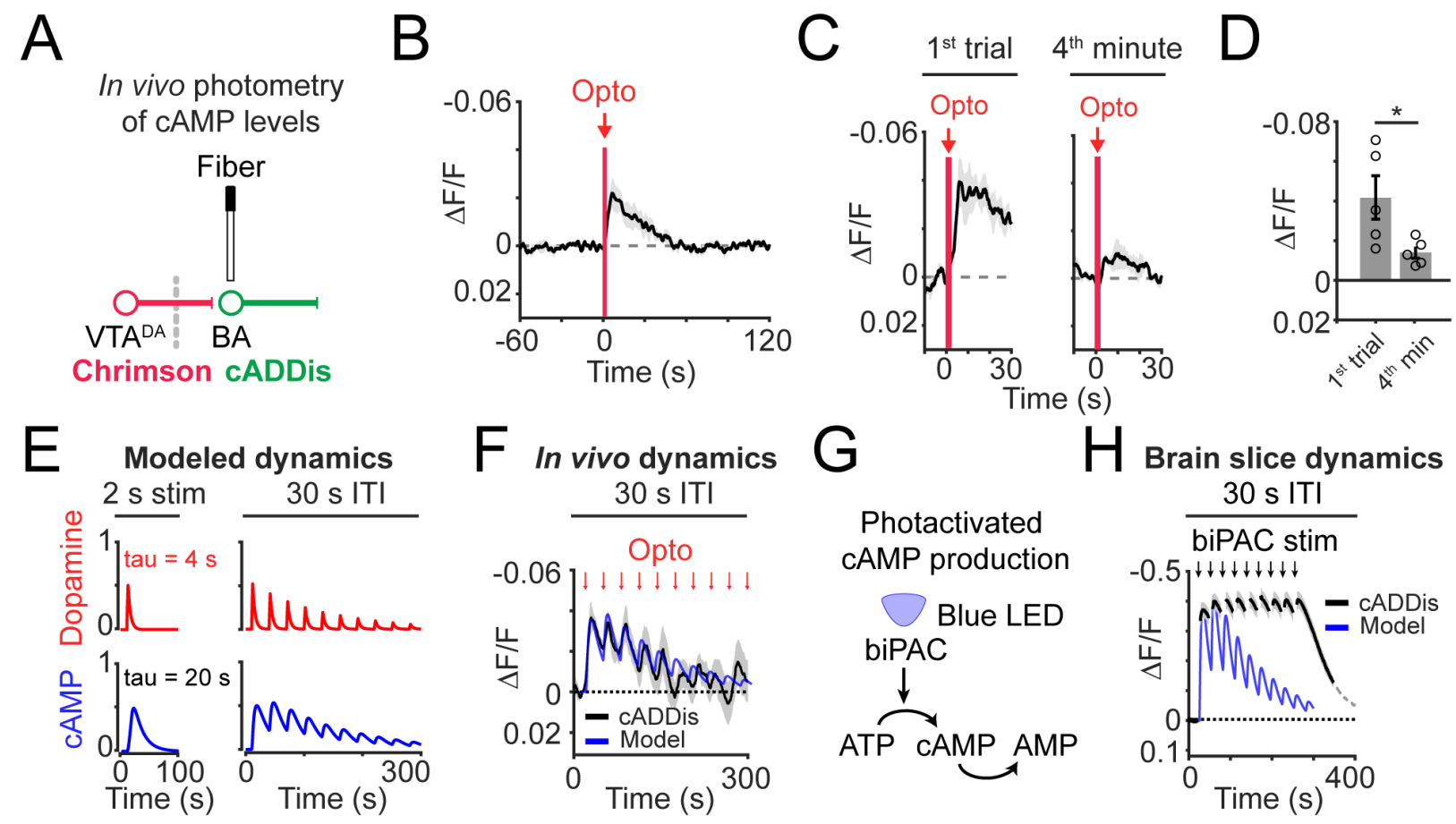




\section{Figure 4}
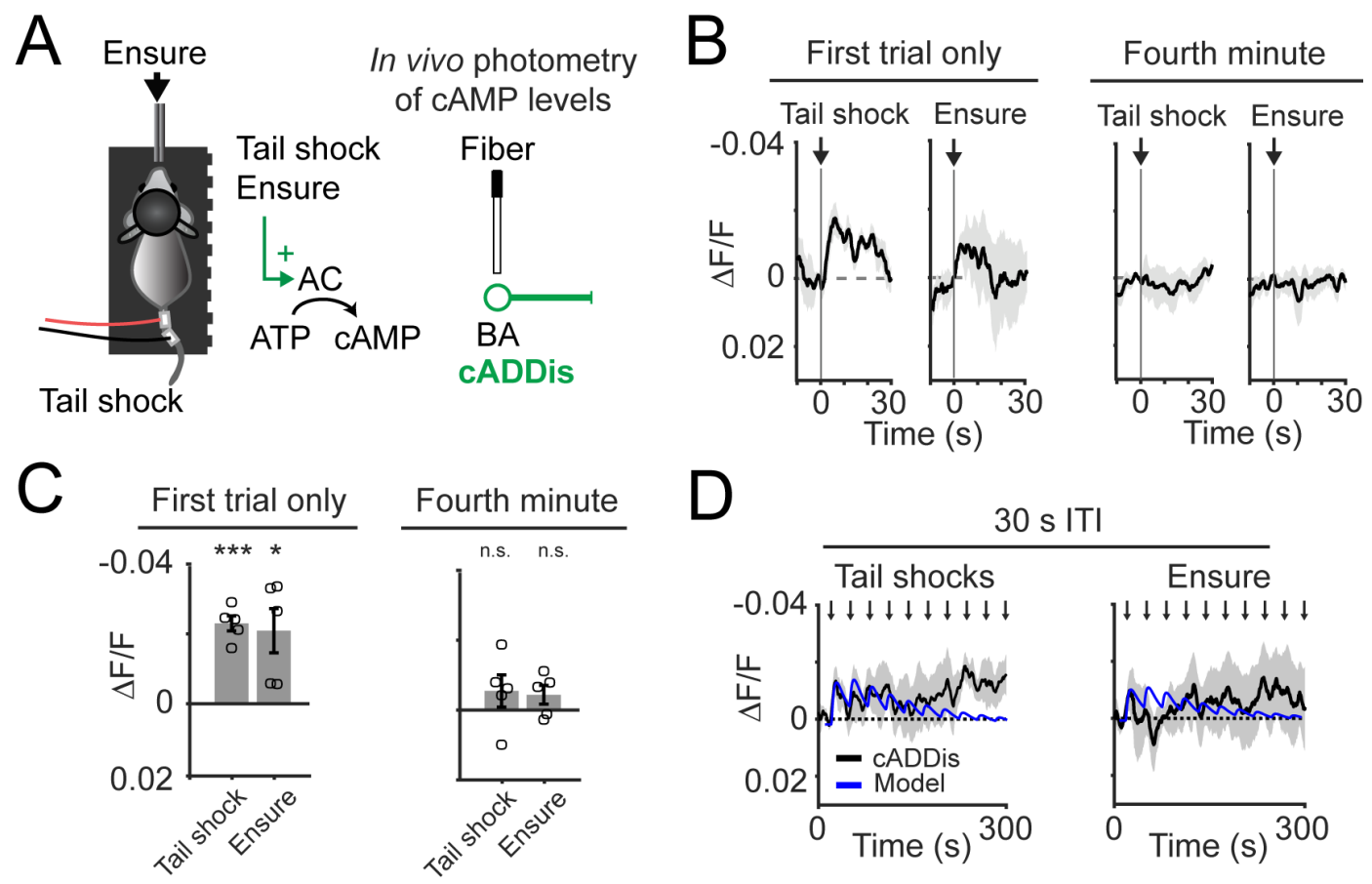

E Tail shock In vivo photometry
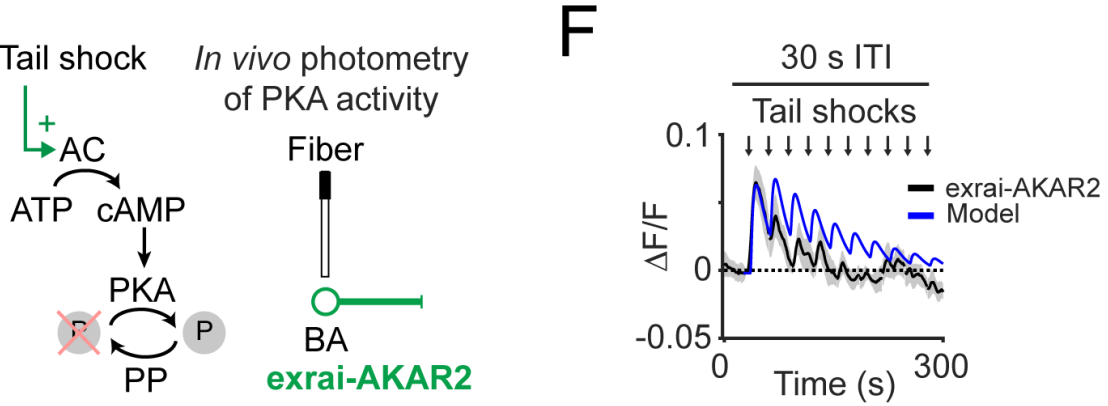


\section{Supplementary Figure 1}

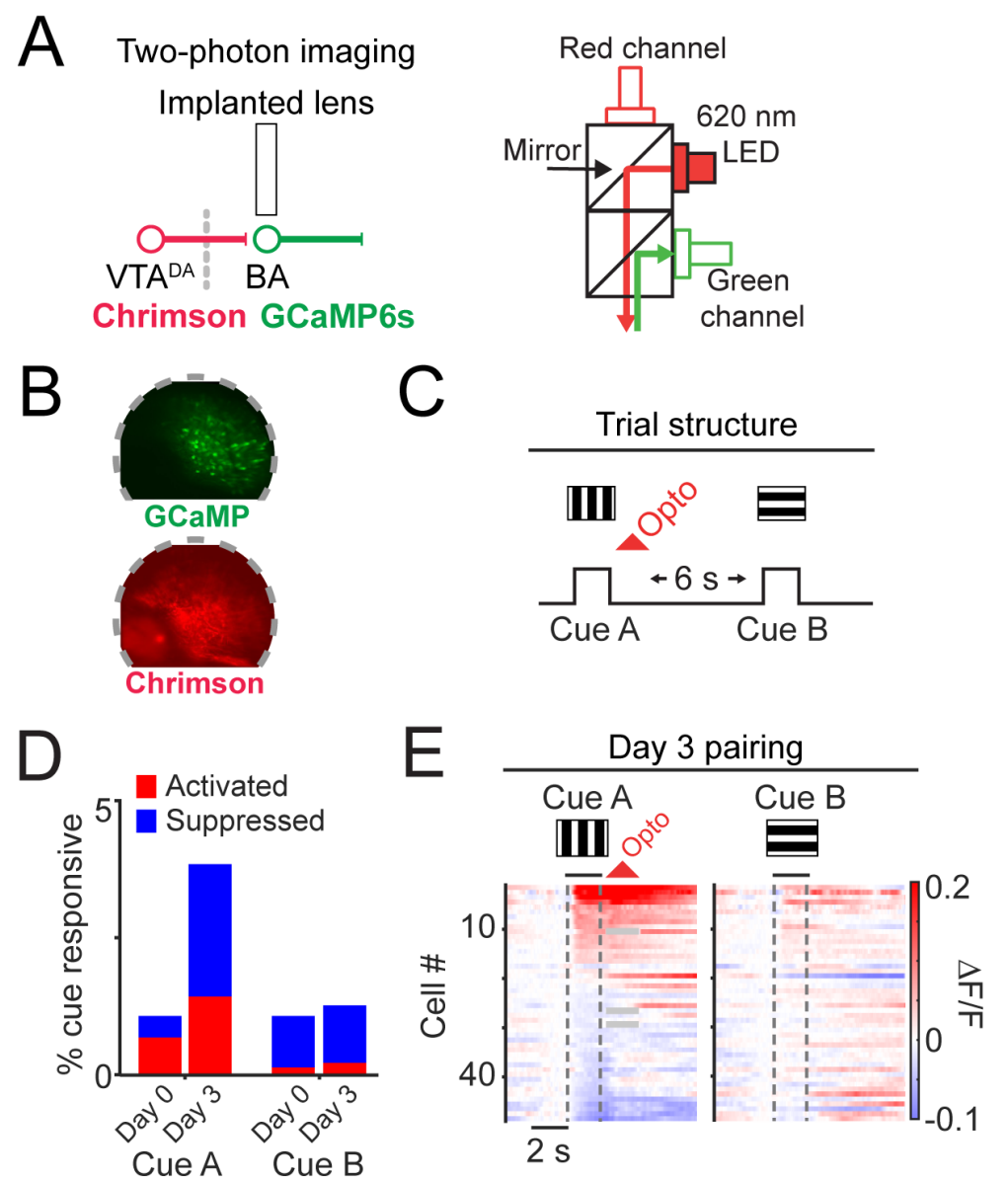

F
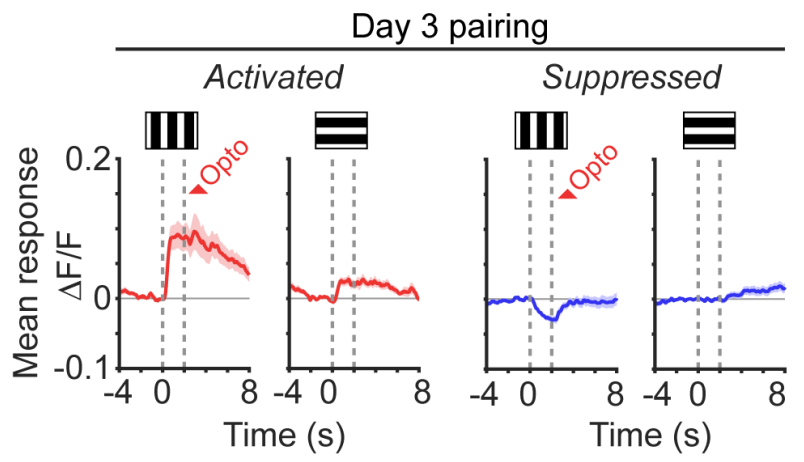

G

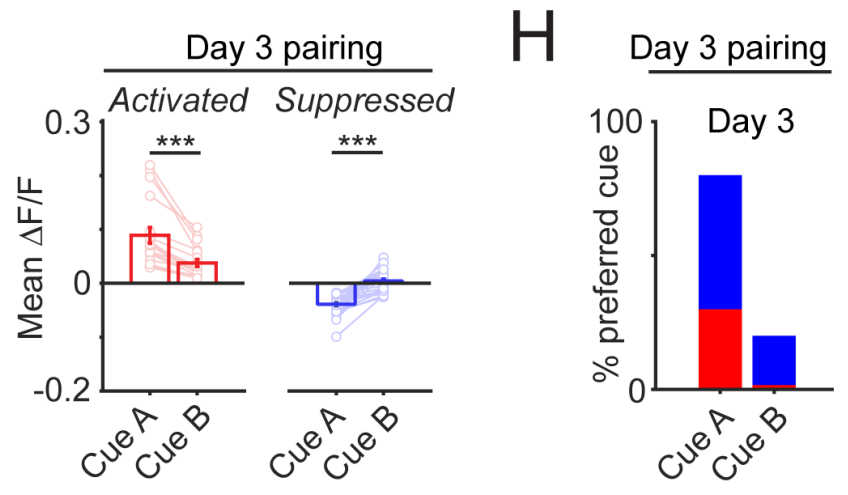




\section{Supplementary Figure 2}

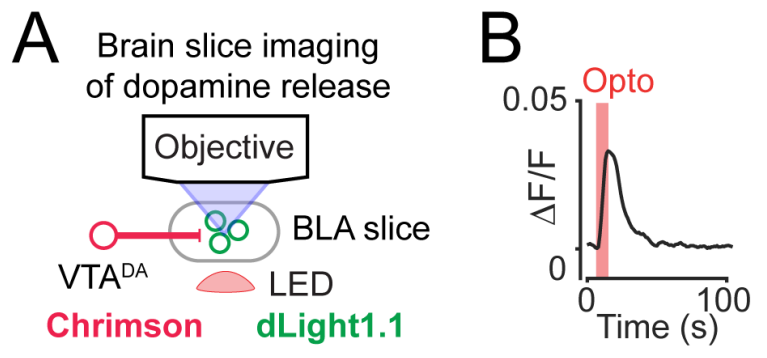

C
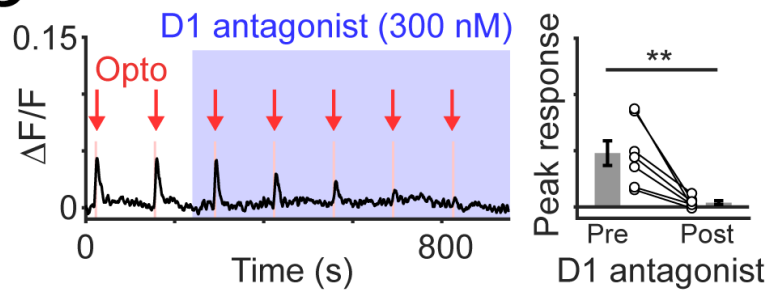

D
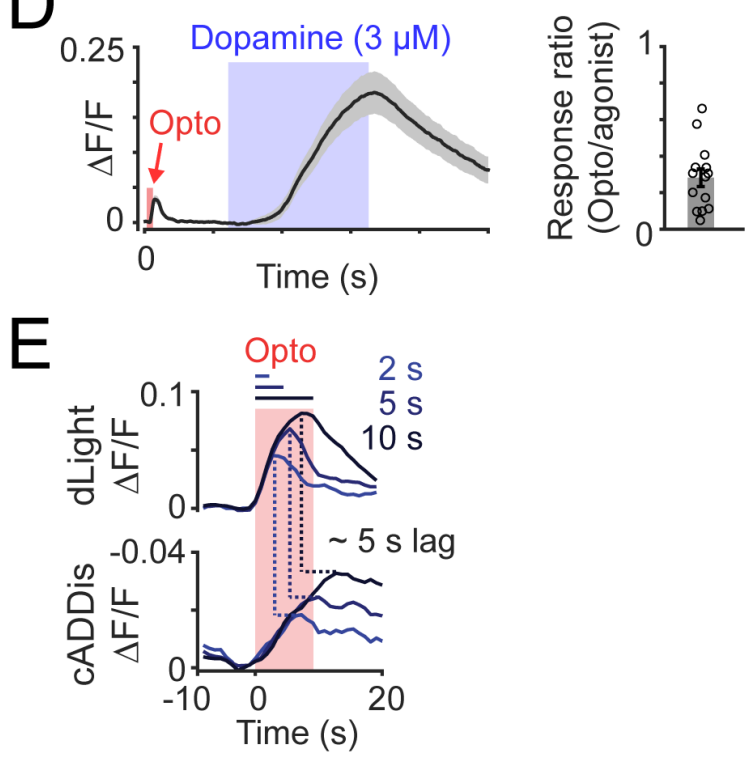


\section{Supplementary Figure 3}

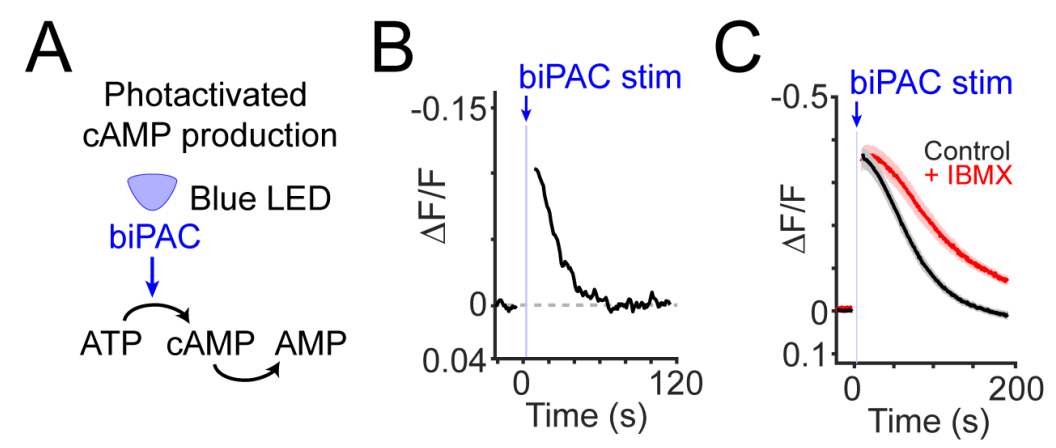




\section{Supplementary Figure 4}

A Intraperitoneal

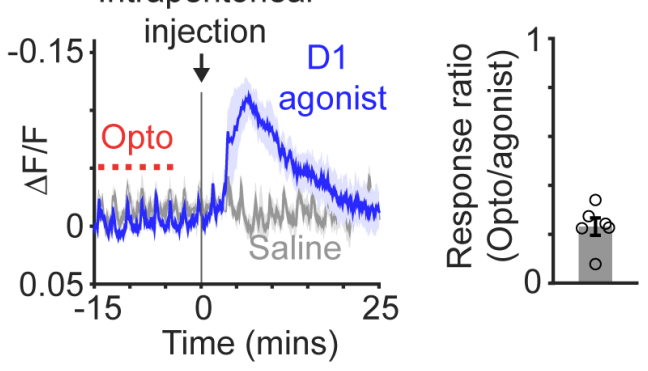

B

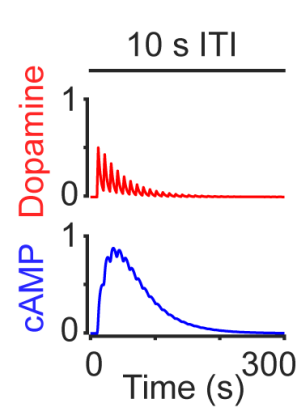

C $10 \mathrm{sITI}$
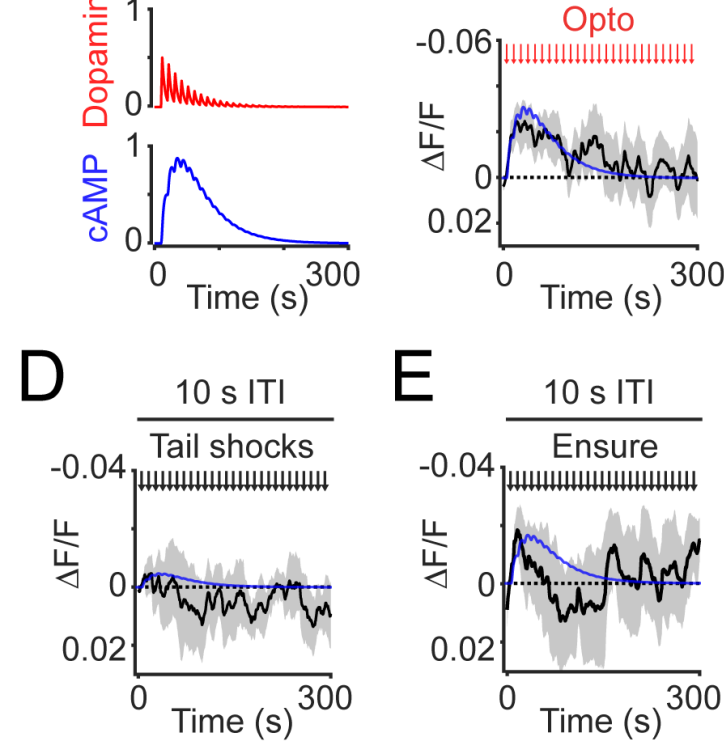


\section{Lead contact}

Further information and requests for resources and reagents should be directed to and will be fulfilled by the lead contact, Mark Andermann (manderma [at] bidmc.harvard.edu).

\section{Materials availability}

For inquiries regarding cADDis plasmid and virus generated during this study, please contact Montana Molecular.

mKate2-biPAC plasmid DNA generated during this study and another study (Zhang et al., 2021) will be deposited to Addgene [catalog \# 169127].

\section{Code and data availability}

Analysis scripts were written in Matlab and used built-in functions and previously published code. No novel code was generated for the purpose of this study. Data is available upon request.

\section{EXPERIMENTAL MODEL AND SUBJECT DETAILS}

\section{Animals}

All animal care and experimental procedures were approved by the Institutional Animal Care and Use Committee at Beth Israel Deaconess Medical Center. Animals were housed in a 12-hourlight/12-hour-darkness environment with standard mouse chow and water provided ad libitum, unless specified otherwise. For both in vitro and in vivo experiments, adult male and female mice between the ages of 9 to 20 weeks were used for this study.

\section{METHOD DETAILS}

\section{Stereotaxic surgeries}


Viral injections, fiber implantations, and GRIN lens implantations were performed as described in (Lutas et al., 2019) with the following specifications and modifications.

For targeting expression into BA glutamatergic neurons, mice (8 - 12 weeks of age) expressing Cre recombinase driven by the Emx1 promoter (EMX1-Cre) were injected at (relative to Bregma): anteroposterior (AP): - $1.6 \mathrm{~mm}$; dorsoventral (DV): $-4.8 \mathrm{~mm}$; mediolateral $(\mathrm{ML}): \pm 3.3 \mathrm{~mm}$. For targeting expression into VTA dopamine neurons, DAT-Cre mice were injected at AP: - $3.2 \mathrm{~mm}$; DV: $-4.5 \mathrm{~mm} ; \mathrm{ML}: \pm 0.4 \mathrm{~mm}$.

For in vivo experiments using the cAMP sensor cADDis, we unilaterally injected AAV1-hSyn-DIOGreenDownward-cADDis (Children's Hospital Vector Core; $300 \mathrm{~nL}$ ). For cADDis or dLight1.1 (AAV1-hSyn-dLight1.1; Children's Hospital Vector Core; $300 \mathrm{~nL}$ ) brain slice imaging experiments, injections were targeted bilaterally to increase the number of useable slices. For in vivo calcium imaging experiments, we unilaterally injected AAV1-hSyn-FLEX-GCaMP6s (Addgene; 300 nL).

For fiber photometry experiments, optic fibers ( $400 \mu \mathrm{m}$ diameter core; multimode; NA 0.48; 5.0 mm length; Doric Fibers) were implanted over BA at (relative to Bregma): AP: $-1.6 \mathrm{~mm}$; DV: -4.7 $\mathrm{mm}$; ML: $3.3 \mathrm{~mm}$.

For in vivo BA cell body imaging of GCaMP6s, mice were implanted with a singlet gradient index (GRIN) lens (GRINtech, NEM-100-25-10-860-S-1.0p; 1.0 mm diameter; 9 mm length; $250 \mu \mathrm{m}$ focal distance on brain side at $860 \mathrm{~nm}$, (NA 0.5); $100 \mu \mathrm{m}$ focal distance on air side (NA 0.5); noncoated). GRIN lens implantation coordinates for cell body imaging of BA neurons in EMX1-Cre transgenic mice (relative to Bregma): AP: $-1.6 \mathrm{~mm}$, ML: $3.2 \mathrm{~mm}$, DV: $-4.8 \mathrm{~mm}$. In order to ensure a snug fit for the lens to reduce brain motion and to increase surgical survival rate, we pre-set the 
insertion tracks by lowering a syringe needle with a slightly narrower diameter (20-gauge, 0.9 $\mathrm{mm}$ ) to a depth of $0.1 \mathrm{~mm}$ above the final depth of the lens.

\section{Widefield epifluorescence imaging of acute brain slices}

Following 3 - 5 weeks of expression, acute slices were prepared as described in Lutas et al., 2019, and widefield fluorescence imaging was performed on an upright microscope (Axioskop 2 plus; Zeiss) equipped with an sCMOS II camera (Prime, Photometrics). Fluorescence excitation for imaging was achieved using a $470 \mathrm{~nm}$ LED (Thorlabs). Image acquisition was performed using ImageJ Micro-manager (Edelstein et al., 2014). Image acquisition frame rate was $2 \mathrm{~Hz}$ for cADDis fluorescence imaging. A 10x (Olympus) or 20x (Zeiss) objective was used for all imaging experiments. During imaging, slices were continuously superfused (flow rate: $2-5 \mathrm{ml} / \mathrm{min}$ ) with oxygenated $\left(95 \% \mathrm{O}_{2}\right.$ and $\left.5 \% \mathrm{CO}_{2}\right)$ artificial cerebrospinal fluid (ACSF) at room temperature. To prevent oxidation of dopamine, $50 \mu \mathrm{M}$ Na-metabisulfite was included in all ACSF solutions during dopamine application experiments.

\section{General two-photon imaging methods}

Two-photon imaging was performed using a two-photon resonant-galvo scanning microscope (NeuroLabWare) at 15.5 frames/second and $796 \times 512$ pixels/frame as described previously (Lutas et al., 2019). An InSight X3 laser (Spectra-Physics) was used to excite the fluorophores (910-1050 nm), and the emission light was filtered (green: 510/84 nm; red: 607/70 nm; Semrock) before collection with photomultiplier tubes (H10770B-40; Hamamatsu). The XY scanning was performed using resonant/galvo mirrors and the $Z$ scanning was achieved with an electrically-tunable lens (Optotune).

\section{Two-photon imaging of acute brain slices}


For two-photon imaging of acute brain slices, slices were prepared as described in Lutas et al. and transferred to a recording chamber perfused with ACSF (oxygenated with $95 \% \mathrm{O}_{2}$ and $5 \% \mathrm{CO}_{2}$; flow rate: $2-5 \mathrm{~mL} / \mathrm{min}$ ) at either room temperature or $32^{\circ} \mathrm{C}$ as indicated. Imaging was performed with a 16x 0.8 NA water-immersion objective (Nikon). The excitation wavelength used was $920 \mathrm{~nm}$.

In optogenetic experiments involving Chrimson, stimulation was triggered by the onset of a frames $(15.5 \mathrm{~Hz})$ for 2-, 5-, or 10-second duration. The gating property of the PMT was triggered at the onset of the frame to protect the PMT from optogenetic stimulation light and lasted for 10 ms. Thus, the top $\sim 16 \%$ of each frame during the stimulation was blank, but much of the frame ( $84 \%)$ provided near-simultaneous imaging of biosensor signals during the photostimulation. A $620 \mathrm{~nm}$ LED ( $1 \mathrm{~mW} / \mathrm{mm}^{2}$, Luxeon Star LEDs) driven by an Arduino-controlled driver (Luxeon Star LEDs) was used for photostimulation.

In optogenetic experiments involving biPAC, each slice was imaged for 10.5 minutes (15.5 frames per second). At time points: $0.5 \mathrm{~min}, 2.5 \mathrm{~min}, 4.5 \mathrm{~min}, 6.5 \mathrm{~min}$ the PMT was powered off, a $470 \mathrm{~nm}$ LED ( $1 \mathrm{~mW} / \mathrm{mm}^{2}$, Luxeon Star LEDs) driven by an Arduino-controlled driver (Luxeon Star LEDs) was then turned on for 2 seconds, and the PMT power was the restored to the original level. The PMT was turned off and on to protect it from the LED light.

To block phosphodiesterase activity, IBMX (100 $\mu \mathrm{M}$ in DMSO) was applied to the brain slice for at least 10 minutes. Recordings of cADDis following biPAC stimulation were performed before and after application of IBMX. To block dLight1.1 signals (dLight1.1 is engineered from the D1 receptor), we applied an antagonist of the D1 receptor (SCH23390; $300 \mathrm{nM})$ while recording

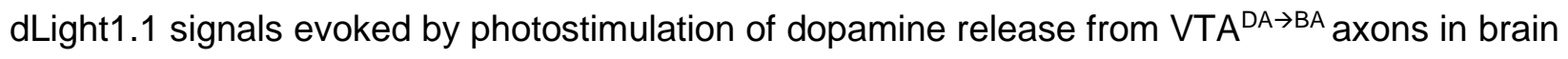
slices.

\section{In vivo two-photon imaging via implanted lens}


Two-photon imaging via implanted lenses was performed as previously described (Lutas et al., 2019) with the following adjustments. A 10x 0.5 NA air objective was used (ThorLabs). For optogenetic stimulation of Chrimson via the implanted lens, a $617 \mathrm{~nm}$ LED (ThorLabs) was used (5 - $10 \mathrm{~mW}$ at the objective face).

\section{Fiber photometry recording}

Fiber photometry recordings were performed as described in Lutas et al., 2019, using head-fixed mice that were free to run on a circular treadmill. Fiber optic cables ( $1 \mathrm{~m}$ long; $400 \mu \mathrm{m}$ core; $0.48 \mathrm{NA}$; Doric Lenses) were coupled to implanted optic fibers with zirconia sleeves (Precision Fiber Products). Excitation and emission light was passed through a four-port fluorescence mini-cube (FMC4_E(460-490)_F(500-550)_O(580-650)_S, Doric Lenses), which allowed for collection of GFP fluorescence and excitation of red-shifted channelrhodopsins. For biosensor photometry recordings, the excitation light $(\sim 100 \mu \mathrm{W}$ at the face of the patch cord) was provided by a $465 \mathrm{~nm}$ LED (Plexon LED and driver). For optogenetic stimulation, the excitation light ( $5 \mathrm{~mW}$ at the face of the patch cord) was provided by a $620 \mathrm{~nm}$ LED (Plexon LED and driver) which was controlled by an Arduino Uno. Emission light was collected by a femtowatt photoreceiver (Newport 2151), demodulated using a lock-in amplifier (SR830; Stanford Instruments) and digitized at $1 \mathrm{kHz}$ sample rate (PCle-6321; National Instruments). Data acquisition was controlled using a custom script in MATLAB (MathWorks). In a subset of experiments the D1 receptor agonist (SKF81297; $20 \mathrm{mg} / \mathrm{kg}$ in saline; Tocris R\&D systems), was injected intraperitoneally while recording cADDis photometry signals. In these experiments, endogenous dopamine release was achieved with Chrimson photostimulation of VTA ${ }^{D A} \rightarrow B A$ terminals and we compared peak cAMP responses following photostimulation and injection of SKF81297. Delivery of appetitive (Ensure) or aversive (tail shock; $0.3 \mathrm{~mA}, 50 \mathrm{~ms}$ ) outcomes was performed as previously described (Lutas et al., 2019). 


\section{QUANTIFICATION AND STATISTICAL ANALYSIS}

\section{Statistics}

The numbers of samples in each group were based on those in previously published studies. Experiments were conducted by an investigator with knowledge of the animal genotype and treatment. Custom-written MATLAB analysis scripts allowed for data analysis in an automated and unbiased manner. All virus expression, optic fiber implants, and GRIN lens placements were verified by post hoc histology. Mice in which either the virus expression or optic fiber was not appropriately located $(<10 \%$ of the time) were excluded from analysis. All data presented as bar and line graphs indicate mean \pm s.e.m. with individual data points also plotted. Statistical analyses were performed in MATLAB. Significance levels are indicated as follows unless otherwise specified: ${ }^{*} p<0.05 ;{ }^{* *} p<0.01 ;{ }^{* * *} p<0.001$.

\section{Data analysis}

All data analyses were performed using MATLAB (Mathworks) and ImageJ (NIH).

\section{Fiber photometry analysis}

Photometry signals were sampled at $1 \mathrm{kHz}$, low pass filtered below $100 \mathrm{~Hz}$, and downsampled to $50 \mathrm{~Hz}$. We calculated $\Delta \mathrm{F} / \mathrm{F}=(\mathrm{F}-\mathrm{F} 0) / \mathrm{F} 0$, where $\mathrm{F} 0$ the mean of baseline window prior to the event of interest. For analysis of the responses to an individual event, all trials containing presentations of that event during a run were averaged to obtain a mean timecourse, and then the peak response during the event window was obtained. We also did not use the sliding-window method for baseline normalized to avoid distorting the slow dynamics of intracellular cAMP signals.

\section{Widefield fluorescence brain slice imaging analysis}


Movies were initially corrected for $x-y$ motion using identical methods (efficient subpixel registration to averaged reference image) as used for two-photon imaging analyses above. For cADDis cAMP sensor imaging, as cells were very bright at baseline (as evoked cAMP leads to a decrease in fluorescence of this sensor) and showed slower dynamic changes in fluorescence upon dopamine application, regions-of-interests were automatically segmented by using morphological filters to identify bright spherical regions (Liang et al., 2018). Briefly, a mean projection through the movie was first applied to obtain a single mean image. Local normalization was then applied by subtracting the local mean (Gaussian kernel with sigma $=8$ pixels) and dividing by local variance across pixels (Gaussian kernel with sigma $=150$ pixels). Basic MATLAB functions were then used to remove small unconnected structures and fill in gaps in larger structures. The image was then binarized and regions of interest were segmented by applying a Euclidian distance transform followed by a watershed transform (a common strategy used to segment spherical objects). We estimated neuropil signals by taking circular annuli around the region of interest, as described above for two-photon image analysis.

\section{Two-photon brain slice and in vivo imaging analysis}

Image registration for brain slice and in vivo two-photon calcium imaging of BA cell bodies was performed as previously described (Lutas et al., 2019). Briefly, to corrected for x-y motion, each frame from an imaging session was registered to a reference image (average of 1000 frames within a session) using efficient subpixel registration methods (Bonin et al., 2011). For extraction of signal from cell body regions of interest (ROIs) from volumetric brain slice imaging (15 depths; $10 \mu \mathrm{m}$ apart), we used CellPose (Stringer et al., 2020), which optimally identified ROls from bright cADDis expressing cell bodies. For in vivo calcium imaging analysis, we used PCA/ICA to extract masks of pixels with correlated activity, corresponding to individual axons or cell bodies (Mukamel et al., 2009). Timecourses were extracted by averaging each of the pixels within each binarized mask. We calculated neuropil activity as the median value of an annulus surrounding 
each ROI (inner radius: 15 pixels; outer radius: 50 pixels; pixels belonging to any other ROI were excluded from these annulus masks). This timecourse of neuropil activity was then subtracted from the activity timecourse of the associated $\mathrm{ROI}$ to create a fluorescence timecourse, $\mathrm{F}(\mathrm{t})$, where $t$ is time of each imaging frame. The change in fluorescence was calculated by subtracting a running estimate of baseline fluorescence $(F 0(t))$ from $F(t)$, then dividing by $F 0(t): \Delta F / F(t)=(F(t)$ - $F 0(t)) / F O(t)$, where $F 0(t)$ is a running estimate of baseline fluorescence calculated as the $10^{\text {th }}$ percentile of $F(t)$ in the previous 32-second sliding window (Petreanu et al., 2012).

\section{Criteria for determining responsivity to cues from in vivo calcium imaging}

To determine if a cell was responsive to each cue, we used previously established, conservative criteria (Lutas et al., 2019), which are described here. We performed a Wilcoxon sign-rank test for each frame post-stimulus onset against the 1-s baseline period prior to stimulus onset, with Bonferroni correction for multiple comparisons across frames $(p<0.01)$. If three consecutive frames were significantly different than the baseline period, a cell was considered responsive to that cue. For all cells with significant responses to at least one cue, a cell preferred cue was determined as the cue evoking the largest response during the cue period. For estimation of a cell's mean cue-evoked response magnitude, and for estimation of a cell's response bias to a given cue, we averaged all trials containing presentations of that cue during the run to obtain a mean timecourse for that cell, and then the maximum response during the 2 -s duration of the cue presentation was used as that cell's response magnitude.

\section{Modeling dopamine-evoked cAMP dynamics}

To model the dynamics of dopamine and cAMP, we first fit monoexponential functions to averaged traces of either dLight1.1 or CADDis recordings. We used the decay rate from the dLight transient to first model dopamine dynamics in response to $2 \mathrm{~s}$ square inputs $(30 \mathrm{~s}$ intervals between each input), which exponentially depressed such that the second input was $25 \%$ weaker 
than the first pulse (as observed in our measurements of dopamine synaptic depression). We convolved this input kernel with an exponential (4s time constant; step size of $0.1 \mathrm{~s})$. We then used this convolved waveform which models dopamine with synaptic depression as an input kernal to model the cAMP dynamics (20 s time constant, step size of $0.1 \mathrm{~s}$ ). For qualitative comparison with in vivo recordings, we normalized the modeled cAMP dynamics to the peak of the first evoked response.

\section{References}

Bonin, V., Histed, M. H., Yurgenson, S., \& Reid, R. C. (2011). Local diversity and fine-scale organization of receptive fields in mouse visual cortex. Journal of Neuroscience, 31(50), 18506-18521. https://doi.org/10.1523/JNEUROSCI.2974-11.2011

Edelstein, A. D., Tsuchida, M. A., Amodaj, N., Pinkard, H., Vale, R. D., \& Stuurman, N. (2014). Advanced methods of microscope control using $\mu$ Manager software. Journal of Biological Methods, 1(2), e10. https://doi.org/10.14440/JBM.2014.36

Liang, L., Fratzl, A., Goldey, G., Ramesh, R. N., Sugden, A. U., Morgan, J. L., Chen, C., \& Andermann, M. L. (2018). A Fine-Scale Functional Logic to Convergence from Retina to Thalamus. Cell, 173(6), 1343-1355.e24. https://doi.org/10.1016/j.cell.2018.04.041

Lutas, A., Kucukdereli, H., Alturkistani, O., Carty, C., Sugden, A. U., Fernando, K., Diaz, V., Flores-Maldonado, V., \& Andermann, M. L. (2019). State-specific gating of salient cues by midbrain dopaminergic input to basal amygdala. Nature Neuroscience, 22(11), 1820-1833. https://doi.org/10.1038/s41593-019-0506-0

Mukamel, E. A., Nimmerjahn, A., \& Schnitzer, M. J. (2009). Automated analysis of cellular signals from large-scale calcium imaging data. Neuron, 63(6), 747-760. 
https://doi.org/10.1016/j.neuron.2009.08.009

Petreanu, L., Gutnisky, D. A., Huber, D., Xu, N., O’Connor, D. H., Tian, L., Looger, L., \& Svoboda, K. (2012). Activity in motor-sensory projections reveals distributed coding in somatosensation. Nature, 489(7415), 299-303. https://doi.org/10.1038/nature11321

Stringer, C., Wang, T., Michaelos, M., \& Pachitariu, M. (2020). Cellpose: a generalist algorithm for cellular segmentation. Nature Methods 2020 18:1, 18(1), 100-106. https://doi.org/10.1038/s41592-020-01018-x

Zhang, S. X., Lutas, A., Yang, S., Diaz, A., Fluhr, H., Nagel, G., Gao, S., \& Andermann, M. L. (2021). Hypothalamic dopamine neurons motivate mating through persistent cAMP signalling. Nature 2021, 1-5. https://doi.org/10.1038/s41586-021-03845-0 